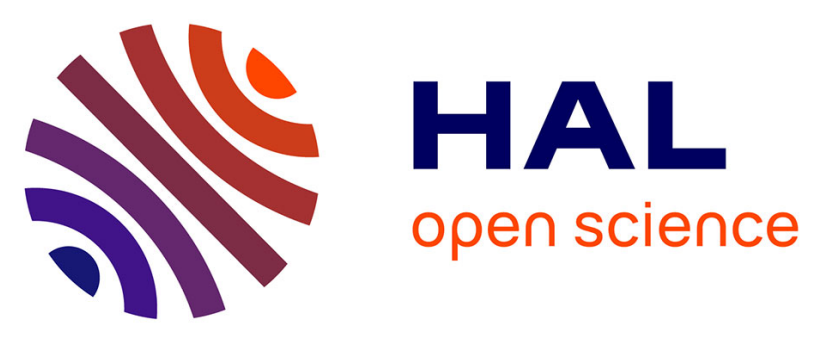

\title{
A compact constitutive model to describe the viscoelastic-plastic behaviour of glassy polymers: Comparison with monotonic and cyclic experiments and state-of-the-art models
}

Thierry Barriere, Xavier Gabrion, Sami Holopainen

\section{To cite this version:}

Thierry Barriere, Xavier Gabrion, Sami Holopainen. A compact constitutive model to describe the viscoelastic-plastic behaviour of glassy polymers: Comparison with monotonic and cyclic experiments and state-of-the-art models. International Journal of Plasticity, 2019, 122, pp.31 - 48. hal-02370579

\author{
HAL Id: hal-02370579 \\ https://hal.science/hal-02370579
}

Submitted on 19 Nov 2019

HAL is a multi-disciplinary open access archive for the deposit and dissemination of scientific research documents, whether they are published or not. The documents may come from teaching and research institutions in France or abroad, or from public or private research centers.
L'archive ouverte pluridisciplinaire HAL, est destinée au dépôt et à la diffusion de documents scientifiques de niveau recherche, publiés ou non, émanant des établissements d'enseignement et de recherche français ou étrangers, des laboratoires publics ou privés. 


\title{
A compact constitutive model to describe the viscoelastic-plastic behaviour of glassy polymers: Comparison with monotonic and cyclic experiments and state-of-the-art models
}

\author{
T. Barriere ${ }^{b}$, X. Gabrion ${ }^{b}$, S. Holopainen ${ }^{\dagger *}$ \\ $\dagger$ Tampere University, Department of Civil Engineering, \\ P.O. Box 600, FI-33014 Tampere, Finland \\ ${ }^{b}$ Univ. Bourgogne Franche-Comté, FEMTO-ST Institute, CNRS/UFC/ENSMM/UTBM, \\ Department of Applied Mechanics, 25000 BESANÇON-FR, France
}

\begin{abstract}
A number of constitutive models have been developed for solid polymers to describe the large deformation behavior. However, most of the existing models rely on a purely elastic or hyperelastic initial response when they are incapable of accurately predicting the cyclic stress-strain hysteresis loops. In this work, therefore, a compact cyclic viscoelastic-viscoplastic constitutive model is proposed to improve the prediction of the loops below the peak yield stress. The proposed approach is based on the distinguished model by Haward and Thackray (1968) for glassy polymers, which is augmented by a few thermodynamically motivated internal state variables able to predict the missing viscous deformation behavior, including the nonlinear cyclic hardening in three dimensions. Based on the comprehensive uniaxial tension experiments, it is demonstrated that this compact formulation, along with a calibration scheme, enables accurate prediction of the shape of the hysteresis loops, as well as the representation of ratcheting behavior. A comparison is also made with state-of-the-art models that are capable of predicting the cyclic stress-strain hysteresis loops.
\end{abstract}

Keywords: Cyclic plasticity, Thermodynamics, Implementation, Experimentation, Ratcheting

\section{Introduction}

Examples of engineering components that are manufactured from polymers and are subjected to cyclic loadings are from fields such as automotive and aerospace systems, marine structures, healthcare instruments, and sporting

*Corresponding author, e-mail: sami.holopainen@tuni.fi 
goods. The components may also be coated by polymers. Concurrently, failures of components due to damage under cyclic long-term loadings have been assessed to be the most significant cause of huge financial losses, Ritchie (1999); Beesley et al. (2017). Despite the importance of polymer components, their cyclic deformation behavior is still under-researched, and much effort is needed to improve their cyclic deformation properties and to develop capable tools for their failure assessment. Considering solid or glassy polymers, the distinguished and recent models are mainly focused on large strain plasticity (group 1), Boyce et al. (1989); Wu and Van der Giessen (1993); Anand and Gurtin (2003); Anand and Ames (2006); Bouvard et al. (2010); Gudimetla and Doghri (2017), or serve a micro-mechanically motivated damage evolution (group 2), Tomita and Uchida (2003); Engqvist et al. (2016); Deng et al. (2017); Jiang et al. (2017); Talamini et al. (2018), or are applied in the research of crack-controlled failure (group 3), Ritchie (1999); James et al. (2013); Ravi Chandran (2016); Awaja et al. (2016); Hughes et al. (2017); Talamini et al. (2018); George et al. (2018). Models in group 2 consider damage processes that emerge in micro-level failures, such as polymer chain breakage and propagation of microscopic flaws termed microvoids and -cracks, Tomita and Uchida (2003); Jiang et al. (2017); Talamini et al. (2018). The research in group 3 is focused on the coalescence of said microflaws when macroscopic cracks may originate and affect the final rupture, James et al. (2013); Ravi Chandran (2016); Awaja et al. (2016); Hughes et al. (2017); Talamini et al. (2018). Most models in group 3 have been implemented to detect crack growth in tiny zones and have not been applied at component level, James et al. (2013); Awaja et al. (2016); Hughes et al. (2017). Only the models proposed in James et al. (2013); Ravi Chandran (2016) are being studied under cyclic loadings, although they are based solely on fatigue life (Wöhler curve). However, owing to the purely elastic or hyperelastic initial response produced by most existing models in groups $1-3$, and by parallel rheological network models in Bengström (2015), the cyclic stress-strain hysteresis loops cannot be well predicted, especially when the stress levels remain below the peak yield stress.

A recent research that is focused on the ratcheting and fatigue behaviors under cyclic loading processes (group 4) can be found from Krairi and Doghri (2014); Jiang et al. (2015); Beesley et al. (2017); Kang and Kan (2017); Shojaei and Volgers (2018); Holopainen and Barriere (2018); Qi et al. (2019), i.e. work on the cyclic deformation behavior of amorphous polymers is very limited. Beesley et al. (2017) introduces an elastic-plastic model to simulate the low-cycle fatigue of notched specimens made of a nickel based alloy. A hyperelastic-viscoplastic model in Shojaei and Volgers (2018) and a parallel rheological network model (elastic-viscoplastic) in Qi et al. (2019) provide capable tools for the investigation of both low and high cycle regimes of a highly-crystalline and semicrystalline polymers, respectively. Considering glassy polymers, however, both the viscoelastic and plastic elements are needed to accurately predict long-term creep/recovery and thereby the cyclic deformation behavior (ratcheting and shape/area of the loops), Anand and Ames (2006); Krairi and Doghri (2014); Jiang et al. (2015). 

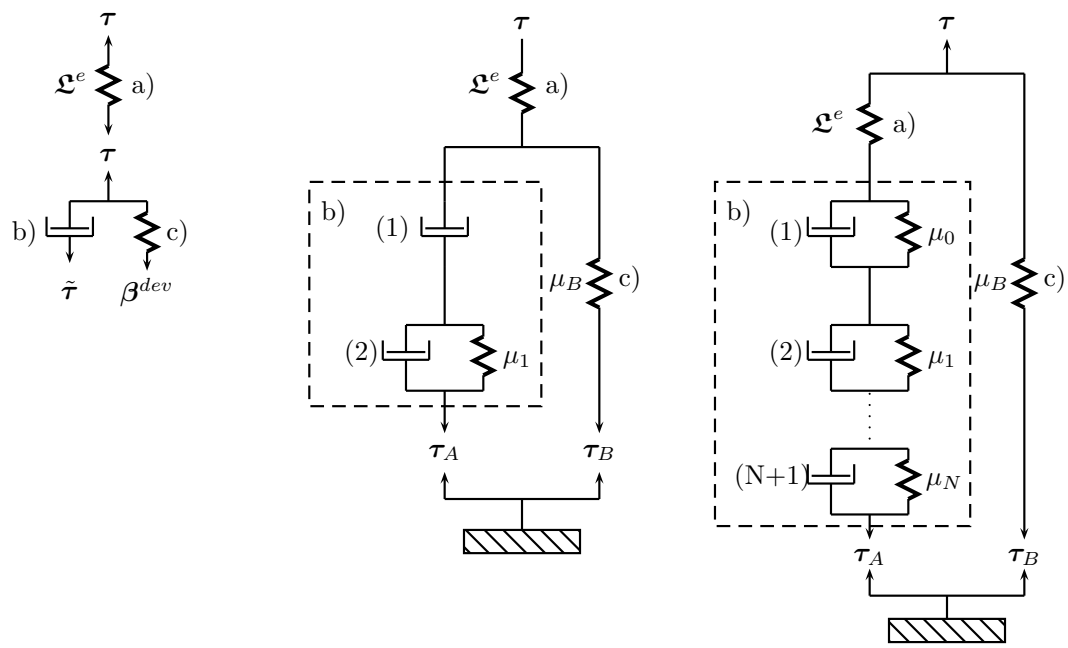

Figure 1: Representation of the three dimensional version of the Haward and Thackray (1968)-model (according to Boyce et al. (1989)), the proposed model, and the Anand and Ames (2006)-model. The models are governed by the following elements: a) an elastic spring, b) a nonlinear system (from a single dashpot to Kelvin-Voigt-like elements, $i=0,1, \ldots, N)$, and c) a nonlinear spring.

This paper studies the modeling of the mechanically motivated cyclic longterm deformation behavior without a remarkable temperature rise and which behavior occurs at relatively small stress frequencies; practically, the stress levels under such conditions remain below the peak yield stress. The paper aims at developing a model capable of predicting more accurately, the shape of the hysteresis loops and amount of energy-dissipation under cyclic loading processes. Another important objective is an exact prediction of the ratcheting behavior including cyclic hardening. Furthermore, the model is suitable for the simulation of very large deformations, i.e. it is based on the decomposition of the deformation gradient into viscoelastic-plastic and purely elastic parts, cf. Boyce et al. (1989); Anand and Gurtin (2003). The formulation of the model follows both the micro-mechanics (anisotropic hardening, Boyce et al. (1989), and yielding including free volume, Anand and Ames (2006)), and internal state variable methodologies (viscoelasticity, Anand and Ames (2006)), and stems from the rheological (spring-dashpot) representations displayed in Fig. 1.

In detail, the proposed approach relies on the pioneering model of Haward and Thackray (1968) for glassy polymers (based on Eyring's theory) that is augmented by a single set of internal variables able to predict the missing viscous deformation behavior (area/shape of the hysteresis loops) and the nonlinear cyclic hardening (ratcheting behavior) in three dimensions. Against the state-of-the-art models of Anand and Gurtin (2003); Anand and Ames (2006); Jiang et al. (2015) that can also predict the cyclic deformation behavior well, the elastic portion of the deformation is described by a single element a) clearly separated from viscoelasticplastic elements in accordance with the classical Haward and Thackray (1968) 
-model. As a result, the nonlinear Langevin spring c) applied in the model to describe the anisotropic hardening of amorphous network structures is modeled solely using the viscous deformation (as originally proposed in Boyce et al. (1989) for three dimension). Moreover, the specified stress ( $\tau_{A}$ in Fig. 1) defines the stress in each micromechanism. Compared with state-of-the-art models, Anand and Ames (2006); Jiang et al. (2015), the proposed model requires a reduced set of internal state variables and material parameters to be defined. The model predictions are evaluated by comparison with the experiments for uniaxial tension under different stress ratios, as well as with the consistent stateof-the-art models' predictions, Boyce et al. (1989); Anand and Ames (2006); Jiang et al. (2015).

This paper is arranged as follows: Kinematics and a thermodynamically consistent constitutive model are introduced subsequently. Section 3 starts with the description of the applied tests, and the calibration procedure for the material parameters is given in Section 3.2. Then, the effectiveness of the approach under creep/recovery and different cyclic loadings for the technologically imperative polymer is discussed in Section 4. Finally, the important conclusions and hotspots for future research are presented.

\section{The approach}

\subsection{Deformation kinematics}

At time $t \in \mathbb{R}^{+}$, deformation of a body $\mathfrak{P}$ is determined by the map $\boldsymbol{y}: \boldsymbol{X} \mapsto$ $\boldsymbol{x}$ in which $\boldsymbol{X} \in \mathfrak{B}$ and $\boldsymbol{x} \in \mathfrak{b}$ denote the material points determined in the undeformed reference and deformed current placements $\mathfrak{B}$ and $\mathfrak{b}$, respectively ${ }^{1}$. The motion of $\mathfrak{b}$ is then given by the map $\boldsymbol{x}=\boldsymbol{y}(\boldsymbol{X}, t)$ and a deformation gradient $\boldsymbol{F}:=\nabla \boldsymbol{y}$, in which $J:=\operatorname{det}(\boldsymbol{F})>0$ is the Jacobian and the operator $\nabla$ denotes the gradient with respect to the point $\boldsymbol{X}$.

The proposed model is founded on the Kröner-Lee decomposition, i.e. the decomposition

$$
\boldsymbol{F}=\boldsymbol{F}^{\mathrm{e}} \boldsymbol{F}^{\mathrm{vep}}
$$

where $\boldsymbol{F}^{\mathrm{e}}$ and $\boldsymbol{F}^{\mathrm{vep}}$ determine the local deformations for the intermediate placement $\overline{\mathcal{N}}$ resulting from elastic and viscoelastic-viscoplastic mechanisms. Whereas the elastic part represents the reversible elastic mechanisms of the chain network, the viscoelastic-plastic part denotes the partially reversible mechanisms of molecular chains, Anand and Gurtin (2003). The viscoelastic-plastic part manifests as long-term creep strain and stress relaxation, due to the inertia of the microstructure to reach its equilibrium. Purely viscoelastic effect appears as recovery of strain after a stress removal, whereas the plastic effect is due to irreversible, dissipative mechanisms, such as chain breakage and the slippage of chain entanglements, Bouvard et al. (2010).

\footnotetext{
${ }^{1}$ The term placement means a certain configuration of a continuum body $\mathfrak{P}$, i.e. the placement is given by $\mathfrak{S}=k(\mathfrak{P})$ under the configuration $k: \mathfrak{P} \rightarrow \mathfrak{S}$, cf. (Zienkiewicz et al., 2014, p. 449). Examples are the reference and current placements, $\mathfrak{B}$ and $\mathfrak{b}$.
} 
The intermediate placement $\overline{\mathcal{N}}$, discussed above, is merely locally determined: $\boldsymbol{F}^{\mathrm{e}}$ and $\boldsymbol{F}^{\mathrm{vep}}$ respectively, being not real deformation gradients. However, $\operatorname{det}\left(\boldsymbol{F}^{\mathrm{e}}\right)>0$ and $\operatorname{det}\left(\boldsymbol{F}^{\mathrm{vep}}\right)>0$ hold, i.e. their inverses exist. Because $\boldsymbol{F}^{\mathrm{e}}$ and $\boldsymbol{F}^{\mathrm{vep}}$ particularize the elastic and viscoelastic-plastic deformations, respectively, they are regarded as uncoupled, and $\overline{\mathcal{N}}$ is stress-free.

Applying the polar decomposition,

$$
\boldsymbol{F}^{\mathrm{e}}=\boldsymbol{v}^{\mathrm{e}} \boldsymbol{R}^{\mathrm{e}}
$$

where $\boldsymbol{v}^{\mathrm{e}}$ is the symmetric stretch tensor and $\boldsymbol{R}^{\mathrm{e}}$ is the rotation associated with the elastic deformation ${ }^{2}$.

The viscoelastic-viscoplastic deformation is defined by a symmetric stretch tensor (in the intermediate placement)

$$
\overline{\boldsymbol{C}}^{\mathrm{vep}}:=\boldsymbol{F}^{\mathrm{vep}} \boldsymbol{F}^{\mathrm{vep}, \mathrm{T}} \text {. }
$$

In (3), the transpose of tensor is symbolized by the letter T. To represent purely elastic deformation, symmetric stretch tensor

$$
\boldsymbol{b}^{\mathrm{e}}:=\boldsymbol{F}^{\mathrm{e}} \boldsymbol{F}^{\mathrm{e}, \mathrm{T}}=:\left(\boldsymbol{v}^{\mathrm{e}}\right)^{2}
$$

given in the spatial placement is also defined. Moreover, the symmetric stretchlike tensors

$$
\overline{\boldsymbol{C}}^{\mathrm{vp}}, \overline{\boldsymbol{C}}^{\mathrm{ve}}
$$

are introduced. These tensors are given in the intermediate placement $\overline{\mathcal{N}}$ characterizing the internal state and its change due to the viscoplastic and viscoelastic deformations, elements (1) and (2) in Fig. 1. They can be viewed as the linear transformations that map vectors in the relaxed space.

\subsection{Rate kinematics}

To model the motion of a solid body, the tensorial velocity gradient

$$
\boldsymbol{l}:=\operatorname{grad}(\mathbf{v})=\dot{\boldsymbol{F}} \boldsymbol{F}^{-1},
$$

in which $\mathbf{v}$ is a velocity field, is defined; grad is the gradient with respect to a deformed point $\boldsymbol{x}$ defined above, and the marker dot means the time derivative. The decomposition (1) in (6) yields

$$
\boldsymbol{l}=\boldsymbol{l}^{\mathrm{e}}+\boldsymbol{l}^{\mathrm{vep}}
$$

where

$$
\boldsymbol{l}^{\mathrm{e}}:=\dot{\boldsymbol{F}}^{\mathrm{e}} \boldsymbol{F}^{\mathrm{e}-1}, \quad \boldsymbol{l}^{\mathrm{vep}}:=\phi_{*}\left(\overline{\boldsymbol{L}}^{\mathrm{vep}}\right), \quad \overline{\boldsymbol{L}}^{\mathrm{vep}}:=\dot{\boldsymbol{F}}^{\mathrm{vep}} \boldsymbol{F}^{\mathrm{vep}-1} .
$$

\footnotetext{
${ }^{2}$ Owing to path dependency of the viscoplastic deformation, observation of stretching and rotations is challenging and thus, the polar decomposition of $\boldsymbol{F}^{\mathrm{vep}}$ is not used.
} 
The operator $\phi_{*}$ in (8) denotes the push-forward operation of the quantity in the parentheses by using $\boldsymbol{F}^{\mathrm{e}}$, cf. (Belytschko et al., 2000, Box 5.16). The specific form of $\phi_{*}$ will be defined in Section 2.3.

Using (3) and (8)(right), the rate of $\overline{\boldsymbol{C}}^{\text {vep }}$ becomes

$$
\dot{\overline{\boldsymbol{C}}}^{\mathrm{vep}}=\overline{\boldsymbol{F}^{\mathrm{vep}} \dot{\boldsymbol{F}}^{\mathrm{vep}, \mathrm{T}}}=\dot{\boldsymbol{F}}^{\mathrm{vep}} \boldsymbol{F}^{\mathrm{vep}, \mathrm{T}}+\boldsymbol{F}^{\mathrm{vep}} \dot{\boldsymbol{F}}^{\mathrm{vep}, \mathrm{T}}=\overline{\boldsymbol{L}}^{\mathrm{vep}} \overline{\boldsymbol{C}}^{\mathrm{vep}}+\overline{\boldsymbol{C}}^{\mathrm{vep}} \overline{\boldsymbol{L}}^{\mathrm{vep}, \mathrm{T}}
$$

Based on (9), (4) in (8) results in

$$
\dot{b}^{\mathrm{e}}=\boldsymbol{l}^{\mathrm{e}} \boldsymbol{b}^{\mathrm{e}}+\boldsymbol{b}^{\mathrm{e}} \boldsymbol{l}^{\mathrm{e}, \mathrm{T}} .
$$

The complements (symmetric, sym, and skew-symmetric, skew) are given by:

$$
\boldsymbol{d}:=\operatorname{sym}(\boldsymbol{l}), \quad \boldsymbol{d}^{\mathrm{e}}:=\operatorname{sym}\left(\boldsymbol{l}^{\mathrm{e}}\right), \quad \boldsymbol{d}^{\mathrm{vep}}:=\operatorname{sym}\left(\boldsymbol{l}^{\mathrm{vep}}\right), \quad \overline{\boldsymbol{D}}^{\mathrm{vep}}:=\operatorname{sym}\left(\overline{\boldsymbol{L}}^{\mathrm{vep}}\right)
$$

and

$\boldsymbol{\omega}:=\operatorname{skew}(\boldsymbol{l}), \quad \boldsymbol{\omega}^{\mathrm{e}}:=\operatorname{skew}\left(\boldsymbol{l}^{\mathrm{e}}\right), \quad \boldsymbol{\omega}^{\mathrm{vep}}:=\operatorname{skew}\left(\boldsymbol{l}^{\mathrm{vep}}\right), \quad \overline{\boldsymbol{W}}^{\text {vep }}:=\operatorname{skew}\left(\overline{\boldsymbol{L}}^{\mathrm{vep}}\right)$,

respectively. It then follows from (7) as follows:

$$
l:=d+\omega=d^{\mathrm{e}}+\boldsymbol{d}^{\mathrm{vep}}+\boldsymbol{\omega}^{\mathrm{e}}+\boldsymbol{\omega}^{\mathrm{vep}}
$$

where $\boldsymbol{d}:=\boldsymbol{d}^{\mathrm{e}}+\boldsymbol{d}^{\mathrm{vep}}$ and $\boldsymbol{\omega}:=\boldsymbol{\omega}^{\mathrm{e}}+\boldsymbol{\omega}^{\mathrm{vep}}$.

As a departure from the previous formulations for polymers, cf. e.g. (Anand and Ames, 2006, eq. (19) with the presumption that the spins vanish therein), it is further defined that

$$
\boldsymbol{l}^{\mathrm{vep}}:=\boldsymbol{l}^{\mathrm{vp}}+\boldsymbol{l}^{\mathrm{ve}}, \boldsymbol{l}^{\mathrm{vp}}:=\boldsymbol{d}^{\mathrm{vp}}+\boldsymbol{\omega}^{\mathrm{vp}}, \boldsymbol{l}^{\mathrm{ve}}:=\boldsymbol{d}^{\mathrm{ve}}+\boldsymbol{\omega}^{\mathrm{ve}} .
$$

\subsection{Thermodynamics of the model}

Thermodynamic treatment as introduced in Anand and Gurtin (2003); Holopainen and Barriere (2018) is used here to describe the dissipative, irreversible and prolonged effects governing the plastic and viscoelastic deformations, respectively. The basis of the theory is the virtual power comprising of two prerequisites:

[1] Power balance: the external and internal virtual powers are equal, i.e. $\mathfrak{W}^{\text {ext }}(\mathfrak{A}, \mathfrak{V})=\mathfrak{W}^{\text {int }}(\mathfrak{A}, \mathfrak{V})$ within a part of the body $\mathfrak{A}(t) \subseteq \mathfrak{b}(t)$ for all virtual velocities $\mathfrak{V}$;

[2] Invariancy under all changes in frame: the internal power $\mathfrak{W}^{\text {int }}(\mathfrak{A}, \mathfrak{V})$ must be frame-indifferent.

In order to treat the force and moment balances (symmetry of stress), which are the consequences of an expenditure of the external virtual power, we refer to previous works, see e.g. Anand and Ames (2006); Holopainen and Barriere (2018). 
Frame-indifference

A theory is required to be invariant under changes in a frame of the space (Euclidean), that considers time-dependent transformations as follows:

$$
\boldsymbol{y}(\boldsymbol{X}, t) \rightarrow \boldsymbol{q}(t)+\boldsymbol{Q}(t)(\boldsymbol{y}(\boldsymbol{X}, t)-\boldsymbol{O})
$$

in which $\boldsymbol{O}$ is a fixed origin, $\boldsymbol{q}(t)$ a vector, and $\boldsymbol{Q}(t)$ is an orthogonal rotation tensor defined at time $t$ for which $\boldsymbol{Q}^{-1}=\boldsymbol{Q}^{\mathrm{T}}$ holds.

Considering the reference placement to be invariant, whereas the current and intermediate placements depend on the choice of changes in frame,

$$
\boldsymbol{F} \rightarrow \boldsymbol{Q F}, \quad \boldsymbol{F}^{\mathrm{vep}} \rightarrow \overline{\boldsymbol{Q}} \boldsymbol{F}^{\mathrm{vep}} \quad \boldsymbol{F}^{\mathrm{e}} \rightarrow \boldsymbol{Q} \boldsymbol{F}^{\mathrm{e}} \overline{\boldsymbol{Q}}^{\mathrm{T}}
$$

where $\boldsymbol{Q}$ and $\overline{\boldsymbol{Q}}$ represent the rotations of the current and intermediate placements. Then, the deformation measures in (3) and (4) are transformed by:

$$
\overline{\boldsymbol{C}}^{\mathrm{vep}} \rightarrow \overline{\boldsymbol{Q}} \overline{\boldsymbol{C}}^{\mathrm{vep}} \overline{\boldsymbol{Q}}^{\mathrm{T}}, \quad \boldsymbol{b}^{\mathrm{e}} \rightarrow \boldsymbol{Q} \boldsymbol{b}^{\mathrm{e}} \boldsymbol{Q}^{\mathrm{T}} .
$$

The transformations (16) in (6) and (8) result in

$$
\begin{aligned}
\boldsymbol{l} & \rightarrow \boldsymbol{Q} \boldsymbol{l} \boldsymbol{Q}^{\mathrm{T}}+\dot{\boldsymbol{Q}} \boldsymbol{Q}^{\mathrm{T}} \text { and } \boldsymbol{d} \rightarrow \boldsymbol{Q} \boldsymbol{d} \boldsymbol{Q}^{\mathrm{T}}, \\
\overline{\boldsymbol{L}}^{\mathrm{vep}} & \rightarrow \overline{\boldsymbol{Q}} \overline{\boldsymbol{L}}^{\mathrm{vp}} \overline{\boldsymbol{Q}}^{\mathrm{T}}+\overline{\overline{\boldsymbol{Q}}} \overline{\boldsymbol{Q}}^{\mathrm{T}}, \quad \overline{\boldsymbol{D}}^{\mathrm{vep}} \rightarrow \overline{\boldsymbol{Q}} \overline{\boldsymbol{D}}^{\mathrm{vp}} \overline{\boldsymbol{Q}}^{\mathrm{T}}, \\
\boldsymbol{l}^{\mathrm{vep}} & \rightarrow \boldsymbol{Q} \boldsymbol{l}^{\mathrm{vep}} \boldsymbol{Q}^{\mathrm{T}}-\boldsymbol{Q} \boldsymbol{F}^{\mathrm{e}-\mathrm{T}} \dot{\bar{Q}}^{\mathrm{T}} \overline{\boldsymbol{Q}} \boldsymbol{F}^{\mathrm{e}-1} \boldsymbol{Q}^{\mathrm{T}}, \\
\boldsymbol{l}^{\mathrm{e}} & \rightarrow \boldsymbol{Q} \boldsymbol{l}^{\mathrm{e}} \boldsymbol{Q}^{\mathrm{T}}+\dot{\boldsymbol{Q}} \boldsymbol{Q}^{\mathrm{T}}+\boldsymbol{Q} \boldsymbol{F}^{\mathrm{e}-\mathrm{T}} \dot{\boldsymbol{Q}}^{\mathrm{T}} \overline{\boldsymbol{Q}} \boldsymbol{F}^{\mathrm{e}-1} \boldsymbol{Q}^{\mathrm{T}} .
\end{aligned}
$$

When leading the results, the property $\overline{\boldsymbol{Q}}^{\mathrm{T}} \dot{\overline{\boldsymbol{Q}}}=-\dot{\overline{\boldsymbol{Q}}}^{\mathrm{T}} \overline{\boldsymbol{Q}}$ has been used. Then, based on (18) $\boldsymbol{l}=\boldsymbol{l}^{\mathrm{e}}+\boldsymbol{l}^{\mathrm{vep}} \rightarrow \boldsymbol{Q}\left(\boldsymbol{l}^{\mathrm{e}}+\boldsymbol{l}^{\mathrm{vep}}\right) \boldsymbol{Q}^{\mathrm{T}}+\dot{\boldsymbol{Q}} \boldsymbol{Q}^{\mathrm{T}}=\boldsymbol{Q} \boldsymbol{l} \boldsymbol{Q}^{\mathrm{T}}+\dot{\boldsymbol{Q}} \boldsymbol{Q}^{\mathrm{T}}$.

Supposing that virtual velocity fields transform as their non-virtual counterparts do, the prerequisite [2] argues that in the current frame, $\mathfrak{W}^{\text {int }}(\mathfrak{A}, \mathfrak{V})=$ $\mathfrak{W}^{\text {int* }}\left(\mathfrak{A}^{*}, \mathfrak{V}^{*}\right)$, where a part of the solid body $\mathfrak{A}$ converts to a region $\mathfrak{A}^{*}$ and $\mathfrak{V}$ to a virtual velocity $\mathfrak{V}^{*}$. Similarly, the stress field $\boldsymbol{\tau}$ transforms to $\boldsymbol{\tau}^{*}$ conjugate to virtual velocities when

$$
\mathfrak{W}^{\text {int }}(\mathfrak{A}, \mathfrak{V}):=\int_{\mathfrak{A}} \boldsymbol{\tau}: \boldsymbol{l} d v=\mathfrak{W}^{\text {int* }}\left(\mathfrak{A}^{*}, \mathfrak{V}^{*}\right):=\int_{\mathfrak{A}}\left(\boldsymbol{\tau}^{*}:\left(\boldsymbol{Q} \tilde{\boldsymbol{l}} \boldsymbol{Q}^{\mathrm{T}}+\dot{\boldsymbol{Q}} \boldsymbol{Q}^{\mathrm{T}}\right) d v\right.
$$

where $\boldsymbol{A}: \boldsymbol{B}:=\operatorname{trace}(\boldsymbol{A} \boldsymbol{B})$. Because the body part $\mathfrak{A}$ is arbitrary, (19) can be localized, i.e. $\boldsymbol{\tau}: \boldsymbol{l}=\boldsymbol{\tau}^{*}:\left(\boldsymbol{Q} \tilde{\boldsymbol{l}} \boldsymbol{Q}^{\mathrm{T}}+\dot{\boldsymbol{Q}} \boldsymbol{Q}^{\mathrm{T}}\right)$. Since the change in frame, $\tilde{\boldsymbol{l}}$, as well as $\dot{Q} \boldsymbol{Q}^{\mathrm{T}}=-\boldsymbol{Q} \dot{\boldsymbol{Q}}^{\mathrm{T}}$ (skew-symmetric) are arbitrary, the stress (symmetric) transforms by $\boldsymbol{\tau} \rightarrow \boldsymbol{Q} \boldsymbol{\tau} \boldsymbol{Q}^{\mathrm{T}}$.

Benefiting (18) in (19) and considering the symmetry of $\boldsymbol{\tau}$ yields

$$
\begin{gathered}
\boldsymbol{\tau}: \boldsymbol{l}^{\mathrm{e}} \rightarrow \boldsymbol{\tau}:\left(\boldsymbol{d}^{\mathrm{e}}+\dot{\boldsymbol{Q}} \boldsymbol{Q}^{\mathrm{T}}\right)+\overline{\boldsymbol{T}}: \dot{\overline{\boldsymbol{Q}}}^{\mathrm{T}} \overline{\boldsymbol{Q}}=\boldsymbol{\tau}: \boldsymbol{d}^{\mathrm{e}}, \\
\boldsymbol{\tau}: \boldsymbol{l}^{\mathrm{vep}} \rightarrow \boldsymbol{\tau}: \boldsymbol{d}^{\mathrm{vep}}+\overline{\boldsymbol{T}}: \overline{\boldsymbol{Q}}^{\mathrm{T}} \dot{\overline{\boldsymbol{Q}}}=\boldsymbol{\tau}: \boldsymbol{d}^{\mathrm{vep}}
\end{gathered}
$$


i.e. $\boldsymbol{\tau}: \boldsymbol{l}=\boldsymbol{\tau}:\left(\boldsymbol{l}^{\mathrm{e}}+\boldsymbol{l}^{\mathrm{vep}}\right)=\boldsymbol{\tau}:\left(\boldsymbol{d}^{\mathrm{e}}+\boldsymbol{d}^{\mathrm{vep}}\right)=\boldsymbol{\tau}: \boldsymbol{d}$ holds. The stress $\overline{\boldsymbol{T}}:=\boldsymbol{F}^{\mathrm{e}-1} \boldsymbol{\tau} \boldsymbol{F}^{\mathrm{e}-\mathrm{T}}$ in (20) is the counterpart of $\boldsymbol{\tau}$ given in the intermediate placement $^{3}$.

\section{Constitutive theory}

The proposed model includes a linear spring a) and a nonlinear spring c) resulting in an initial elastic response and anisotropic effect of material in large deformations, see Fig. 1. To predict increasing macro-yielding followed by a notable softening effect, the model also includes a nonlinear dashpot (1). To improve the predictions of the cyclic hysteresis loops, the model is augmented by a system for viscoelastic micro-mechanisms consisting of a KelvinVoigt-like element (2) arranged in series with the dashpot (1). Moreover, the element (2) governs creep and recovery, and its action together with the spring a) is used to model stress relaxation.

Using the equations (7) and (11), and noting the symmetry of stress $\boldsymbol{\tau}$, the localized dissipation in its spatial form becomes

$$
\mathcal{D}=\boldsymbol{\tau}: \boldsymbol{d}^{\mathrm{e}}+\boldsymbol{\tau}: \boldsymbol{d}^{\mathrm{vep}}-\dot{\psi} \geq 0
$$

where $\mathcal{D}$ is the power of the local dissipation and $\dot{\psi}$ stands for the rate of the Helmholtz free energy per unit volume. The Helmholtz free energy $\psi$ is taken to be a sum of several independent potentials, Chaboche (1997); Anand and Ames (2006), i.e.

$$
\psi=\hat{\psi}\left(\boldsymbol{b}^{\mathrm{e}}, \overline{\boldsymbol{C}}^{\mathrm{vep}}, \overline{\boldsymbol{C}}^{\mathrm{ve}}, \varphi\right)=\hat{\psi}^{\mathrm{e}}\left(\boldsymbol{b}^{\mathrm{e}}\right)+\hat{\psi}^{\mathrm{vep}}\left(\overline{\boldsymbol{C}}^{\mathrm{vep}}\right)+\hat{\psi}^{\mathrm{ve}}\left(\overline{\boldsymbol{C}}^{\mathrm{ve}}, \varphi\right)
$$

wherein $\psi^{\mathrm{e}}, \psi^{\mathrm{vep}}$, and $\psi^{\mathrm{ve}}$ are the contributions associated with the elastic spring a), the nonlinear Langevin spring c), and the nonlinear spring in the viscoelastic element $(2)$, respectively. The viscoelastic potential $\psi^{\mathrm{ve}}$ is also taken to be the function of the free volume, $\varphi^{4}$.

Remark 2.1. To guarantee the invariance of the free energy, finally invariants of the tensor arguments in the potential function (22) will be used.

The rate $\dot{\varphi}$ is given by

$$
\dot{\psi}=\frac{\partial \psi^{\mathrm{e}}}{\partial \boldsymbol{b}^{\mathrm{e}}}: \dot{\boldsymbol{b}}^{\mathrm{e}}+\frac{\partial \psi^{\mathrm{vep}}}{\partial \overline{\boldsymbol{C}}^{\mathrm{vep}}}: \dot{\overline{\boldsymbol{C}}}^{\mathrm{vep}}+\frac{\partial \psi^{\mathrm{ve}}}{\partial \overline{\boldsymbol{C}}^{\mathrm{ve}}}: \dot{\overline{\boldsymbol{C}}}^{\mathrm{ve}}+\frac{\partial \psi^{\mathrm{ve}}}{\partial \varphi} \dot{\varphi}
$$

Taking advantage of (10), noting the symmetry of $\boldsymbol{b}^{\mathrm{e}}$, and considering $\psi^{\mathrm{e}}$ as an isotropic function of $\boldsymbol{b}^{\mathrm{e}}$ result in

$$
\frac{\partial \psi^{\mathrm{e}}}{\partial \boldsymbol{b}^{\mathrm{e}}}: \dot{\boldsymbol{b}}^{\mathrm{e}}=2 \frac{\partial \psi^{\mathrm{e}}}{\partial \boldsymbol{b}^{\mathrm{e}}} \boldsymbol{b}^{\mathrm{e}}: \boldsymbol{d}^{\mathrm{e}}
$$

\footnotetext{
${ }^{3}$ It then follows that the operator $\phi_{*}=\boldsymbol{F}^{\mathrm{e}-\mathrm{T}}(\cdot) \boldsymbol{F}^{\mathrm{e}-1}$ in (8).

${ }^{4}$ Evolution of the local free-volume is considered an important source for the transient peak associated with yielding of amorphous polymers, Anand and Ames (2006).
} 
In accordance with (24) and noting the symmetry of $\overline{\boldsymbol{C}}^{\mathrm{vep}}$ by (9),

$$
\frac{\partial \psi^{\text {vep }}}{\partial \overline{\boldsymbol{C}}^{\text {vep }}}: \dot{\overline{\boldsymbol{C}}}^{\text {vep }}=2 \frac{\partial \psi^{\text {vep }}}{\partial \overline{\boldsymbol{C}}^{\text {vep }}} \overline{\boldsymbol{C}}^{\text {vep }}: \overline{\boldsymbol{D}}^{\text {vep }}=: \overline{\boldsymbol{B}}^{\mathrm{vep}}: \overline{\boldsymbol{D}}^{\mathrm{vep}}=\boldsymbol{\beta}^{\text {vep }}: \boldsymbol{d}^{\text {vep }}
$$

in which $\overline{\boldsymbol{B}}^{\text {vep }}$ represents an internal back stress quantity that controls the anisotropic hardening at large strains owing to the viscous work accumulated in a material body. The spatial complement $\boldsymbol{\beta}^{\mathrm{vep}}$ is derived by the push-forward operation determined in (20) and represents the stress in branch B (Fig. 1), i.e. $\tau^{\mathrm{B}}:=\boldsymbol{\beta}^{\mathrm{vep}}$.

Moreover, using the symmetry of $\overline{\boldsymbol{C}}^{\text {ve }}$ given by (5), one can define:

$$
\frac{\partial \psi^{\mathrm{ve}}}{\partial \overline{\boldsymbol{C}}^{\mathrm{ve}}}: \dot{\overline{\boldsymbol{C}}}^{\mathrm{ve}}=: 2 \frac{\partial \psi^{\mathrm{ve}}}{\partial \overline{\boldsymbol{C}}^{\mathrm{ve}}} \overline{\boldsymbol{C}}^{\mathrm{ve}}: \overline{\boldsymbol{D}}^{\mathrm{ve}}=: \overline{\boldsymbol{B}}^{\mathrm{ve}}: \overline{\boldsymbol{D}}^{\mathrm{ve}}=\boldsymbol{\beta}^{\mathrm{ve}}: \boldsymbol{d}^{\mathrm{ve}}
$$

where $\overline{\boldsymbol{B}}^{\mathrm{ve}}$ is an internal stress acting on the spring in the element (2). Its spatial counterpart in the deformed current placement is $\boldsymbol{\beta}^{\mathrm{ve}}$. Consistent with (26),

$$
\dot{\overline{\boldsymbol{C}}}^{\mathrm{ve}}:=\overline{\boldsymbol{D}}^{\mathrm{ve}} \overline{\boldsymbol{C}}^{\mathrm{ve}}+\overline{\boldsymbol{C}}^{\mathrm{ve}} \overline{\boldsymbol{D}}^{\text {ve }} \text {. }
$$

A complementary dissipation potential, associated with the dashpot (1) and that in the element (2) (cf. Fig. 1), is as

$$
\phi\left(\boldsymbol{\tau}^{(1)}, \boldsymbol{\tau}^{(2)}\right):=\phi^{\mathrm{vp}}\left(\boldsymbol{\tau}^{(1)}\right)+\phi^{\mathrm{ve}}\left(\boldsymbol{\tau}^{(2)}\right)
$$

where $\boldsymbol{\tau}^{(1)}:=\boldsymbol{\tau}_{\mathrm{A}}$ and $\boldsymbol{\tau}^{(2)}:=\boldsymbol{\tau}_{\mathrm{A}}-\boldsymbol{\beta}^{\mathrm{ve}}$ stand for the driving stresses in the dashpots of the elements (1) and (2), respectively.

Benefiting the potential $\phi$, a part of the dissipation power becomes

$$
\mathcal{D}=\frac{\partial \phi^{\mathrm{vp}}}{\partial \boldsymbol{\tau}^{(1)}}: \boldsymbol{\tau}^{(1)}+\frac{\partial \phi^{\mathrm{ve}}}{\partial \boldsymbol{\tau}^{(2)}}: \boldsymbol{\tau}^{(2)} .
$$

Considering (29), substitution of (24), (25), and (26) into (21) results in

$$
\begin{aligned}
& \left(\boldsymbol{\tau}-2 \frac{\partial \psi^{\mathrm{e}}}{\partial \boldsymbol{b}^{\mathrm{e}}} \boldsymbol{b}^{\mathrm{e}}\right): \boldsymbol{d}^{\mathrm{e}}=0, \\
& \left(\boldsymbol{\tau}-\boldsymbol{\beta}^{\mathrm{vep}}\right): \boldsymbol{d}^{\mathrm{vep}}-\boldsymbol{\beta}^{\mathrm{ve}}: \boldsymbol{d}^{\mathrm{ve}}-\frac{\partial \psi^{\mathrm{ve}}}{\partial \varphi} \dot{\varphi}-\frac{\partial \phi^{\mathrm{vp}}}{\partial \boldsymbol{\tau}^{(1)}}: \boldsymbol{\tau}^{(1)}-\frac{\partial \phi^{\mathrm{ve}}}{\partial \boldsymbol{\tau}^{(2)}}: \boldsymbol{\tau}^{(2)}=0 .
\end{aligned}
$$

Equation (30) must be valid under any possible thermodynamically admissible mechanisms, when the constitutive equation

$$
\boldsymbol{\tau}=2 \frac{\partial \psi^{\mathrm{e}}}{\partial \boldsymbol{b}^{\mathrm{e}}} \boldsymbol{b}^{\mathrm{e}}
$$

is obtained. Defining

$$
\dot{\tilde{s}}^{(1)}:=-\frac{\partial \phi^{\mathrm{vp}}}{\partial \boldsymbol{\tau}^{(1)}}: \boldsymbol{\tau}^{(1)} \text { and } \dot{\tilde{s}}^{(2)}:=-\frac{\partial \phi^{\mathrm{ve}}}{\partial \boldsymbol{\tau}^{(2)}}: \boldsymbol{\tau}^{(2)},
$$

which represent the rates of the decreasing internal variables in the dashpots of the elements (1) and (2), (30) 2 can be written in the compact form as:

$$
\boldsymbol{\tau}_{\mathrm{A}}: \boldsymbol{d}^{\mathrm{vep}}=\boldsymbol{\beta}^{\mathrm{ve}}: \boldsymbol{d}^{\mathrm{ve}}+\frac{\partial \psi^{\mathrm{ve}}}{\partial \varphi} \dot{\varphi}-\dot{\tilde{s}}^{(1)}-\dot{\tilde{s}}^{(2)} .
$$


Theorem 2.2. The model satisfies the dissipation (Clausius-Duhem) inequality $\mathcal{D} \geq 0$.

Proof. Considering (31), (32), and (33) in (30), the local dissipation power in (21) becomes:

$$
\mathcal{D}=-\dot{\tilde{s}}^{(1)}-\dot{\tilde{s}}^{(2)} .
$$

Since $\dot{\tilde{s}}^{(1)} \leq 0$ and $\dot{\tilde{s}}^{(2)} \leq 0$ in (34), $\mathcal{D}$ always is greater than or equal to zero

\subsection{Distinct constitutive equations}

\subsubsection{Elasticity}

The approach is refined to address amorphous polymers. The chain network structure of the amorphous polymers is practically disorderly when a usual isotropic strain energy function,

$$
\psi^{\mathrm{e}}=\frac{1}{2} \kappa^{\mathrm{e}}\left(I_{1}^{\mathrm{e}}\right)^{2}+2 \mu^{\mathrm{e}} J_{2}^{\mathrm{e}},
$$

where $\kappa^{\mathrm{e}}$ and $\mu^{\mathrm{e}}$ denote the bulk and the shear moduli, is suitable. The invariants in (35) are given by:

$$
I_{1}^{\mathrm{e}}:=\operatorname{trace}\left(\ln \boldsymbol{v}^{\mathrm{e}}\right)=\ln J^{\mathrm{e}}, J_{2}^{\mathrm{e}}:=\frac{1}{2}\left(\ln \boldsymbol{v}^{\mathrm{e}}\right)^{\operatorname{dev}}:\left(\ln \boldsymbol{v}^{\mathrm{e}}\right)^{\operatorname{dev}},
$$

wherein $J^{\mathrm{e}}:=\operatorname{det}\left(\boldsymbol{v}^{\mathrm{e}}\right)$. The stress equation follows from (31) and (35):

$$
\boldsymbol{\tau}=\mathfrak{L}^{\mathrm{e}}: \ln \boldsymbol{v}^{\mathrm{e}},
$$

where $\mathfrak{L}^{\mathrm{e}}$ is the standard fourth order elasticity tensor, see (Holopainen and Wallin, 2012, eqs. (11,12)),(Holopainen et al., 2017, eq. (47)).

\subsubsection{Inelasticity}

Flow rule

According to (14), the rate of viscoelastic-plastic deformation is given by the sum

$$
\boldsymbol{d}^{\mathrm{vep}}=\boldsymbol{d}^{\mathrm{vp}}+\boldsymbol{d}^{\mathrm{ve}} .
$$

The viscoelastic contribution $\boldsymbol{d}^{\mathrm{ve}}$, associated with the mechanism (2) in Fig. 1, is determined by a rule as:

$$
\boldsymbol{d}^{\mathrm{ve}}:=\dot{\gamma}^{\mathrm{ve}} \boldsymbol{n}^{(2)}, \quad \boldsymbol{n}^{(2)}:=\frac{\boldsymbol{\tau}^{(2), \mathrm{dev}}}{\sqrt{2} \tau^{(2)}}, \quad \tau^{(2)}:=\sqrt{\frac{1}{2} \boldsymbol{\tau}^{(2), \mathrm{dev}}: \boldsymbol{\tau}^{(2), \mathrm{dev}}}
$$

where $\boldsymbol{\tau}^{(2) \text {,dev }}=\boldsymbol{\tau}^{\mathrm{A} \text {,dev }}-\boldsymbol{\beta}^{\mathrm{ve}, \mathrm{dev}}$ and $\dot{\gamma}^{\mathrm{ve}}$ represents a positive, effective viscoelastic strain rate defined subsequently. The deviatoric part dev for each tensor [.] is defined via the identity $\boldsymbol{i}$ as: $[\cdot]^{\text {dev }}:=[\cdot]-1 / 3 \operatorname{trace}([\cdot]) \boldsymbol{i}$.

Consistent with (38), the rate $\boldsymbol{d}^{\mathrm{vp}}$, associated with the mechanism (1), is determined as

$$
\boldsymbol{d}^{\mathrm{vp}}:=\dot{\gamma}^{\mathrm{vp}} \boldsymbol{n}^{(1)}, \quad \boldsymbol{n}^{(1)}:=\frac{\boldsymbol{\tau}^{(1), \mathrm{dev}}}{\sqrt{2} \tau^{(1)}}, \quad \tau^{(1)}:=\sqrt{\frac{1}{2} \boldsymbol{\tau}^{(1), \mathrm{dev}}: \boldsymbol{\tau}^{(1), \mathrm{dev}}}
$$


where $\boldsymbol{\tau}^{(1), \operatorname{dev}}=\boldsymbol{\tau}^{\mathrm{A}, \mathrm{dev}}=\boldsymbol{\tau}^{\mathrm{dev}}-\boldsymbol{\beta}^{\mathrm{vep}, \mathrm{dev}}$ and $\dot{\gamma}^{\mathrm{vp}}$ represents a positive, effective viscoplastic strain rate. It then follows from (38) in (27) that

$$
0=\operatorname{trace}\left(\boldsymbol{d}^{\mathrm{ve}}\right)=\operatorname{trace}\left(\overline{\boldsymbol{D}}^{\mathrm{ve}}\right)=\operatorname{trace}\left(\overline{\boldsymbol{C}}^{\mathrm{ve}-\mathrm{T}} \dot{\overline{\boldsymbol{C}}}^{\mathrm{ve}, \mathrm{T}}\right)=\frac{1}{\operatorname{det}\left(\overline{\boldsymbol{C}}^{\mathrm{ve}}\right)} \frac{d}{d t} \operatorname{det}\left(\overline{\boldsymbol{C}}^{\mathrm{ve}}\right)
$$

or $\operatorname{det}\left(\overline{\boldsymbol{C}}^{\text {ve }}\right)=1$ (equals to the undeformed value). Similarly, using (9) and noting $(37), \operatorname{det}\left(\overline{\boldsymbol{C}}^{\text {vep }}\right)=1$, i.e. the viscoelastic-plastic stretching is incompressible when $J^{\mathrm{e}}:=\operatorname{det}\left(\boldsymbol{F}^{\mathrm{e}}\right)=: J$.

\section{Plastic deformation}

Plastic deformation, which governs the irreversible mechanisms of the material, is considered the most dominant in amorphous solid polymers. Physically motivated Boyce et al. (1989)-model and Anand and Ames (2006)-model represent two approaches capable of governing the development of the plastic deformation through the element (1) in Fig. 1. Because the Anand and Ames (2006)-model can predict the transient yield peak that is typical to amorphous polymers more accurately, this model is used in this study.

The evolving plastic deformation through the dashpot (1) is modeled by the following power-law type strain rate

$$
\dot{\gamma}^{\mathrm{vp}}=\dot{v}_{0}\left(\frac{\tau^{(1)}}{s^{(1)}+\alpha p}\right)^{1 / \mathrm{m}_{0}}
$$

where $\dot{v}_{0}, m_{0}$, and $\alpha$ denote the material parameters, and $p=-\operatorname{trace}\left(\boldsymbol{\tau}_{\mathrm{A}} / J^{\mathrm{e}}\right) / 3$ is the pressure. Strain softening is modeled by an internal variable as:

$$
\dot{s}^{(1)}=h_{0} \dot{\gamma}^{\mathrm{vp}}\left(1-s^{(1)} / \tilde{s}^{(1)}(\varphi)\right), \quad s^{(1)}(0)=s_{0}
$$

where

$$
\tilde{s}^{(1)}:=s_{\mathrm{Sv}}\left(1+b\left(\varphi_{\mathrm{sv}}-\varphi\right)\right)
$$

with

$$
\dot{\varphi}:=g_{0} \dot{\gamma}^{\mathrm{vp}}\left(\frac{s^{(1)}}{s_{\mathrm{Sv}}}-1\right), \quad \varphi(0)=0
$$

for evolving free volume. Additional parameters needed are $h_{0}, s_{0}, g_{0}, s_{\mathrm{sv}}, b$, and $\varphi_{\mathrm{sv}}$.

\section{Anisotropic hardening}

To model the anisotropic hardening behavior in large strains, several choices for the plastic portion of the free energy are available. The most prominent ones are based on the 8-chain and full network models, cf. Boyce et al. (1989); $\mathrm{Wu}$ and Van der Giessen (1993) and Fig. 2.

According to the Anand and Ames (2006)-model, the anisotropic deformation behavior in large strains is based on the entire deformation (spring c) in Fig. 1). However, as discussed in Introduction, this treatment can be questioned for 

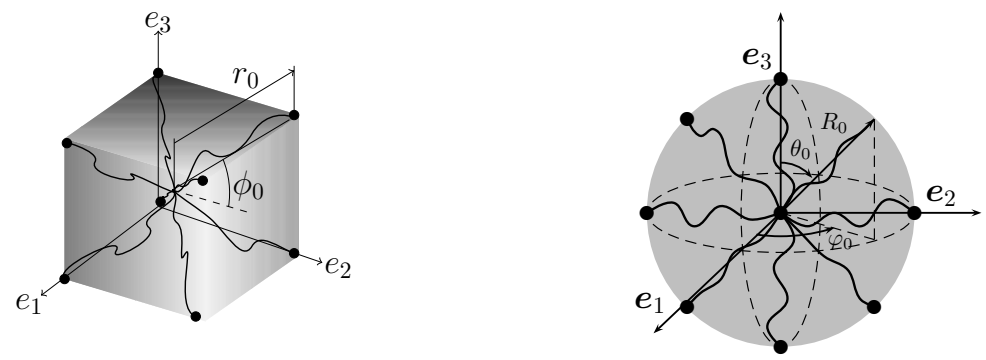

Figure 2: Idealized chain structure according to the 8-chain model (left) and the full network model (right). The dimension of the 8-chain cube is given by $r_{0}$ and $\phi_{0}$, and $R_{0}$ denotes the radius of the micro-sphere.

amorphous solid polymers, and the idea that the anisotropic deformation behavior is solely given in terms of the viscoelastic-plastic response is applied in the proposed model. Based on the 8-chain modeling concept, cf. Boyce et al. (1989), the viscoelastic-plastic portion of the free energy in the proposed model (the element c) in Fig. 1) is governed by the function:

$$
\psi^{\mathrm{vep}}\left(\lambda_{\mathrm{ec}}^{\mathrm{vep}}\right)=C_{R}\left(\frac{\lambda_{\mathrm{ec}}^{\mathrm{vep}}}{\sqrt{N}} \xi+\ln \left(\frac{\xi}{\sinh (\xi)}\right)\right) \geq 0, \quad \psi^{\mathrm{vep}}(1)=0
$$

wherein $C_{R}$ and $N$ stand for parameters, and $\xi:=\mathfrak{L}^{-1}\left(\lambda_{\mathrm{ec}}^{\mathrm{vep}} / \sqrt{N}\right)$ means the inverse of the Langevin function, $\mathfrak{L}$. Applying a non-affine network stretch

$$
\lambda_{\mathrm{ec}}^{\mathrm{vep}}:=\frac{1}{\sqrt{3}} \sqrt{\operatorname{trace}\left(\overline{\boldsymbol{C}}^{\mathrm{vep}}\right)},
$$

as has been done in Boyce et al. (1989), the backstress $\overline{\boldsymbol{B}}^{\text {vep }}$ representing kinematic hardening becomes

$$
\overline{\boldsymbol{B}}^{\mathrm{vep}}=\frac{C^{\mathrm{R}}}{3 \lambda_{\mathrm{ec}}^{\mathrm{ve}}} \sqrt{N} \xi \overline{\boldsymbol{C}}^{\mathrm{vep}} .
$$

When calculating $\xi$ in (47), the Padé approximation is applied, cf. Cohen (1991).

Corollary 2.3. The spatial counterpart $\boldsymbol{\beta}^{\mathrm{vep}}$ of $\overline{\boldsymbol{B}}^{\mathrm{vep}}$ takes the form

$$
\boldsymbol{\beta}^{\mathrm{vep}}=\frac{C^{\mathrm{R}}}{3 \lambda_{\mathrm{ec}}^{\mathrm{vep}}} \sqrt{N} \xi c^{\mathrm{vep}},
$$

where $\boldsymbol{c}^{\mathrm{vep}}=J^{\mathrm{e}-2 / 3} \boldsymbol{F}^{\mathrm{e}} \overline{\boldsymbol{C}}^{\mathrm{vep}} \boldsymbol{F}^{\mathrm{e}, \mathrm{T}}$.

Proof. Considering the kinetic, stress measures contravariant, cf. (Belytschko et al., 2000, Box 5.16), $\overline{\boldsymbol{C}}^{\mathrm{vep}}$ in (47) is in contravariant form. Then the push-forward operation (determined in connection with $(20)$ ) of $\overline{\boldsymbol{C}}^{\text {vep }}$ results in $\hat{\boldsymbol{c}}^{\text {vep }}:=$ $\boldsymbol{F}^{\mathrm{e}} \overline{\boldsymbol{C}}^{\mathrm{vep}} \boldsymbol{F}^{\mathrm{e}, \mathrm{T}}$. Using (38) and (39) in (37) reveals that $\boldsymbol{d}^{\mathrm{vep}}$ is deviatoric, when

$0=\operatorname{trace}\left(\boldsymbol{d}^{\mathrm{vep}}\right)=\operatorname{trace}\left(\overline{\boldsymbol{D}}^{\mathrm{vep}}\right)=\operatorname{trace}\left(\overline{\boldsymbol{C}}^{\mathrm{vep}-\mathrm{T}} \dot{\overline{\boldsymbol{C}}}^{\mathrm{vep}, \mathrm{T}}\right)=\frac{1}{\operatorname{det}\left(\overline{\boldsymbol{C}}^{\mathrm{vep}}\right)} \frac{d}{d t} \operatorname{det}\left(\overline{\boldsymbol{C}}^{\mathrm{vep}}\right)$,

i.e. $\operatorname{det}\left(\boldsymbol{F}^{\mathrm{e}-1} \hat{\boldsymbol{c}}^{\mathrm{vep}} \boldsymbol{F}^{\mathrm{e}-\mathrm{T}}\right)=\operatorname{det}\left(\boldsymbol{F}^{\mathrm{e}-1}\right) \operatorname{det}\left(\hat{\boldsymbol{c}}^{\mathrm{vep}}\right) \operatorname{det}\left(\boldsymbol{F}^{\mathrm{e}-\mathrm{T}}\right)=1$. It is then required that $\hat{\boldsymbol{c}}^{\mathrm{vep}}=J^{\mathrm{e}+2 / 3} \boldsymbol{c}^{\mathrm{vep}}$ 
Viscoelastic deformation

Consistent with (41) for viscoplasticity, the viscoelastic deformation through the dashpot in element (2) is governed by

$$
\dot{\gamma}^{\mathrm{ve}}=\dot{v}_{0}\left(\frac{\tau^{(2)}}{s^{(2)}+\alpha p}\right)^{1 / \mathrm{m}_{1}} \geq 0
$$

where $m_{1}$ is a material parameter. Against the internal variable $s^{(1)}$ defined above, $s^{(2)}$ is regarded as a constant parameter.

The back stress influencing the viscoelastic element is defined by the function of the neo-Hookean form as follows:

$$
\psi^{\mathrm{ve}}=\frac{3}{2} \mu_{1}\left(\left(\lambda_{\mathrm{ec}}^{\mathrm{ve}}\right)^{2}-1\right),
$$

where

$$
\lambda_{\mathrm{ec}}^{\mathrm{ve}}:=1 / \sqrt{3} \sqrt{\operatorname{trace}\left(\overline{\boldsymbol{C}}^{\mathrm{ve}}\right)}
$$

is an effective viscoelastic stretch, and the back stress modulus $\mu_{1}:=\hat{\mu}_{1}(\varphi)$ is considered a function of the local free volume $\varphi$ as

$$
\dot{\mu}_{1}:=c_{1}\left(1-\frac{\mu_{1}}{\mu_{1, \text { sat }}}\right) \dot{\varphi}, \quad \mu_{1}(0)=\mu_{1}^{0}
$$

involving the material parameters $c_{1}, \mu_{1, \text { sat }}$, and $\mu_{1}^{0}$. Expecting $\mu_{1, \text { sat }} \leq \mu_{1}^{0}, \mu_{1}$ reduces to its saturation value $\mu_{1 \text {,sat }}$ as the free-volume increases. Then, in view of (51),

$$
\frac{\partial \psi^{\mathrm{ve}}}{\partial \overline{\boldsymbol{C}}^{\mathrm{ve}}}=\frac{\mu_{1}}{2} \overline{\boldsymbol{I}}
$$

where $\overline{\boldsymbol{I}}$ is the identity given in the intermediate placement, gives

$$
\overline{\boldsymbol{B}}^{\mathrm{ve}}:=\mu_{1} \overline{\boldsymbol{C}}^{\mathrm{ve}} .
$$

Benefiting Corollary 2.3, the spatial form becomes $\boldsymbol{\beta}^{\mathrm{ve}}:=\mu_{1} \boldsymbol{c}^{\mathrm{ve}}$, where $\boldsymbol{c}^{\mathrm{ve}}=$ $J^{\mathrm{e}-2 / 3} \boldsymbol{F}^{\mathrm{e}} \overline{\boldsymbol{C}}^{\mathrm{ve}} \boldsymbol{F}^{\mathrm{e}, \mathrm{T}}$.

The treatment of the dissipation inequality (21) based on the constitutive equations is given in Appendix A. A numerical solution method is introduced in Appendix B. A brief summary of the approach is presented in Table 1.

\section{Experimentation}

An amorphous thermoplastic polycarbonate (PC) (Lexan ${ }^{\circledR}$ 223R granulate) was used to calibrate and evaluate the proposed model. Dogbone-shaped specimens for both monotonic and cyclic loading tests were injected in accordance

with the standard ASTM D638-03 with the type IV specimen, ASTM Committee (2003),Barriere et al. (2018). The geometry of the specimen is demonstrated in Fig. 3. 
Table 1: Summary of the proposed model.

1. Kinematics: $\boldsymbol{F}=\boldsymbol{F}^{\mathrm{e}} \boldsymbol{F}^{\mathrm{vep}}, \boldsymbol{b}^{\mathrm{e}}=\boldsymbol{F}^{\mathrm{e}} \boldsymbol{F}^{\mathrm{e}, \mathrm{T}}, \boldsymbol{v}^{\mathrm{e}}=\sqrt{\boldsymbol{b}^{\mathrm{e}}}, \overline{\boldsymbol{C}}^{\mathrm{vep}}:=\boldsymbol{F}^{\mathrm{vep}} \boldsymbol{F}^{\mathrm{vep}, \mathrm{T}}, \boldsymbol{c}^{\mathrm{vep}}=J^{\mathrm{e}-2 / 3} \phi_{*}\left(\overline{\boldsymbol{C}}^{\mathrm{vep}}\right), \overline{\boldsymbol{C}}^{\mathrm{ve}}$, $\boldsymbol{c}^{\mathrm{ve}}=J^{\mathrm{e}-2 / 3} \phi_{*}\left(\overline{\boldsymbol{C}}^{\mathrm{ve}}\right), \overline{\boldsymbol{D}}^{\mathrm{ve}}=\phi^{*}\left(\boldsymbol{d}^{\mathrm{ve}}\right), \boldsymbol{d}^{\mathrm{vep}}=\boldsymbol{d}^{\mathrm{vp}}+\boldsymbol{d}^{\mathrm{ve}}, \overline{\boldsymbol{D}}^{\mathrm{vep}}=\phi^{*}\left(\boldsymbol{d}^{\mathrm{vep}}\right), \overline{\boldsymbol{L}}^{\mathrm{vep}}=\overline{\boldsymbol{D}}^{\mathrm{vep}}+\overline{\boldsymbol{W}}^{\mathrm{vep}}$.

2. Kinetics: $\quad \boldsymbol{\tau}=\mathfrak{L}^{\mathrm{e}}: \ln \boldsymbol{v}^{\mathrm{e}}=2 \mu\left(\ln \boldsymbol{v}^{\mathrm{e}}\right)^{d e v}+\kappa \ln J^{e} \boldsymbol{i}, \quad \boldsymbol{\tau}_{B}=\frac{C_{R}}{3 \lambda_{\mathrm{ec}}^{\mathrm{vep}}} \sqrt{N} \mathfrak{L}^{-1}\left(\frac{\lambda_{\mathrm{ec}}^{\mathrm{vep}}}{\sqrt{N}}\right) \boldsymbol{c}^{\mathrm{vep}}$ (anisotropic hardening), $\tau_{\mathrm{A}}=\boldsymbol{\tau}-\tau_{\mathrm{B}}, \quad \boldsymbol{\beta}^{\mathrm{vep}}:=\tau_{\mathrm{B}}$.

$2.1 \quad$ Micro-macro transition: $\quad \lambda_{\text {ec }}^{\mathrm{vep}}=\frac{1}{\sqrt{3}} \sqrt{\left(\overline{\boldsymbol{I}}: \overline{\boldsymbol{C}}^{\mathrm{vep}}\right)} \in[0, \sqrt{N}]$.

3. Viscoelastic and viscoelastic-plastic micromechanisms:

$3.1 \quad$ Flow rules: $\quad \boldsymbol{d}^{\mathrm{vep}}=\boldsymbol{d}^{\mathrm{vp}}+\boldsymbol{d}^{\mathrm{ve}}=\dot{\gamma}^{\mathrm{vp}} \boldsymbol{n}^{(1)}+\dot{\gamma}^{\mathrm{ve}} \boldsymbol{n}^{(2)}, \quad \boldsymbol{n}^{(1)}:=\frac{\boldsymbol{\tau}_{\mathrm{A}}^{\mathrm{dev}}}{\sqrt{2} \tau_{\mathrm{A}}^{\mathrm{dev}}}, \quad \tau_{\mathrm{A}}^{\mathrm{dev}}:=\sqrt{\frac{1}{2} \boldsymbol{\tau}_{\mathrm{A}}^{\mathrm{dev}}: \boldsymbol{\tau}_{\mathrm{A}}^{\mathrm{dev}}}$, $\boldsymbol{n}^{(2)}:=\frac{\boldsymbol{\tau}_{\mathrm{A}}^{\mathrm{dev}}-\boldsymbol{\beta}^{\mathrm{ve}}}{\sqrt{2} \tau^{(2)}}, \quad \tau^{(2)}:=\sqrt{\frac{1}{2}\left(\boldsymbol{\tau}_{\mathrm{A}}^{\mathrm{dev}}-\boldsymbol{\beta}^{\mathrm{ve}}: \boldsymbol{\tau}_{\mathrm{A}}^{\mathrm{dev}}-\boldsymbol{\beta}^{\mathrm{ve}}\right)}$

3.2 Loading, unloading (viscoelasticity): $\quad \dot{\gamma}^{\mathrm{ve}}:=\dot{v}_{0}\left(\frac{\tau^{(2)}}{s^{(2)}+\alpha p}\right)^{\frac{1}{m_{1}}}, s^{(2)}=s^{(2)}(0), \quad \boldsymbol{\beta}^{\mathrm{ve}}=\mu_{1} \boldsymbol{c}^{\mathrm{ve}}$, $\dot{\mu}_{1}:=c_{1}\left(1-\frac{\mu_{1}}{\mu_{1, \text { sat }}}\right) \dot{\varphi}, \quad \mu_{1}(\varphi(0))=\mu_{1}^{0}>\mu_{1, \text { sat }}$.

3.3 Yielding, softening (viscoplasticity): $\dot{\varphi}=g_{0}\left(\frac{s^{(1)}}{s_{\mathrm{cv}}}-1\right) \dot{\gamma}^{\mathrm{vp}}, \varphi(0)=\varphi_{0}, \quad \dot{\gamma}^{\mathrm{vp}}:=\dot{v}_{0}\left(\frac{\tau_{\mathrm{A}}^{\mathrm{dev}}}{s^{(1)}+\alpha p}\right)^{\frac{1}{m_{0}}}$, $\left.p=-\frac{1}{3} \operatorname{trace}\left(\boldsymbol{\tau}_{A}\right), \dot{s}^{(1)}=h_{0}\left(1-\frac{s^{(1)}}{\tilde{s}^{(1)}}\right) \dot{\gamma}^{\mathrm{vp}}, \tilde{s}^{(1)}(\varphi)\right):=s_{\mathrm{sv}}\left(1+b\left(\varphi_{\mathrm{sv}}-\varphi\right)\right), \varphi(0)<\varphi_{c v}$.

3.5 Evolution of kinematical variables: $\quad \dot{\boldsymbol{F}}^{\mathrm{vep}}:=\overline{\boldsymbol{L}}^{\mathrm{vep}} \boldsymbol{F}^{\mathrm{vep}}, \dot{\overline{\boldsymbol{C}}}^{\mathrm{ve}}:=\overline{\boldsymbol{D}}^{\mathrm{ve}} \overline{\boldsymbol{C}}^{\mathrm{ve}}+\overline{\boldsymbol{C}}^{\mathrm{ve}} \overline{\boldsymbol{D}}^{\mathrm{ve}}$.

Model parameters: $\dot{v}_{0}, \alpha, s^{(2)}, m_{1}, c_{1}, \mu_{1, \mathrm{sat}}, \mu_{1}^{0}$ (viscoelasticity), $s_{0}, m_{0}, C_{\mathrm{R}}, N, h_{0}, g_{0}, b, s_{\mathrm{cv}}, \varphi_{\mathrm{cv}}, \varphi_{0}$ (viscoplasticity).

\subsection{Uniaxial tensile tests}

The experiments were conducted under isothermal conditions (room temperature and low stress/strain rates) without a marked temperature rise (below 10 $\left.{ }^{\circ} \mathrm{C}\right)$, i.e. the tests are considered quasi-static. An Instron Electropulse E10000 machine equipped with a $10 \mathrm{kN}$ load sensor was used. An extensometer with the gauge length of $25 \mathrm{~mm}$ was also applied to measure the axial elongation $u$, see Fig. 3. The strain was defined by $\epsilon:=u / L$, where $L$ is the gauge length of the extensometer used. The stress was defined by $\sigma:=F / A$, where $F$ is the force (measured by the machine) and the area of the initial cross section is given by $A^{5}$. The monotonic tensile tests were performed at different strain rates (displacement control) $\dot{\epsilon}=0.001 \mathrm{~s}^{-1}, \dot{\epsilon}=0.01 \mathrm{~s}^{-1}$, and $\dot{\epsilon}=0.1 \mathrm{~s}^{-1}$. The acquisition frequency for these tests was $20 \mathrm{~Hz}$.

\footnotetext{
${ }^{5}$ The measured stress is actually the 1st Piola Kirchoff stress, Belytschko et al. (2000); the Kirchhoff stress applied in the model is $\tau:=\sigma \lambda$, where $\sigma$ is the Cauchy/true stress and $\lambda=1+\epsilon$ is the axial stretch. However, the difference between the Kirchhoff, Cauchy, and 1st Piola Kirchoff stress is small under the strains $\epsilon<0.3$.
} 

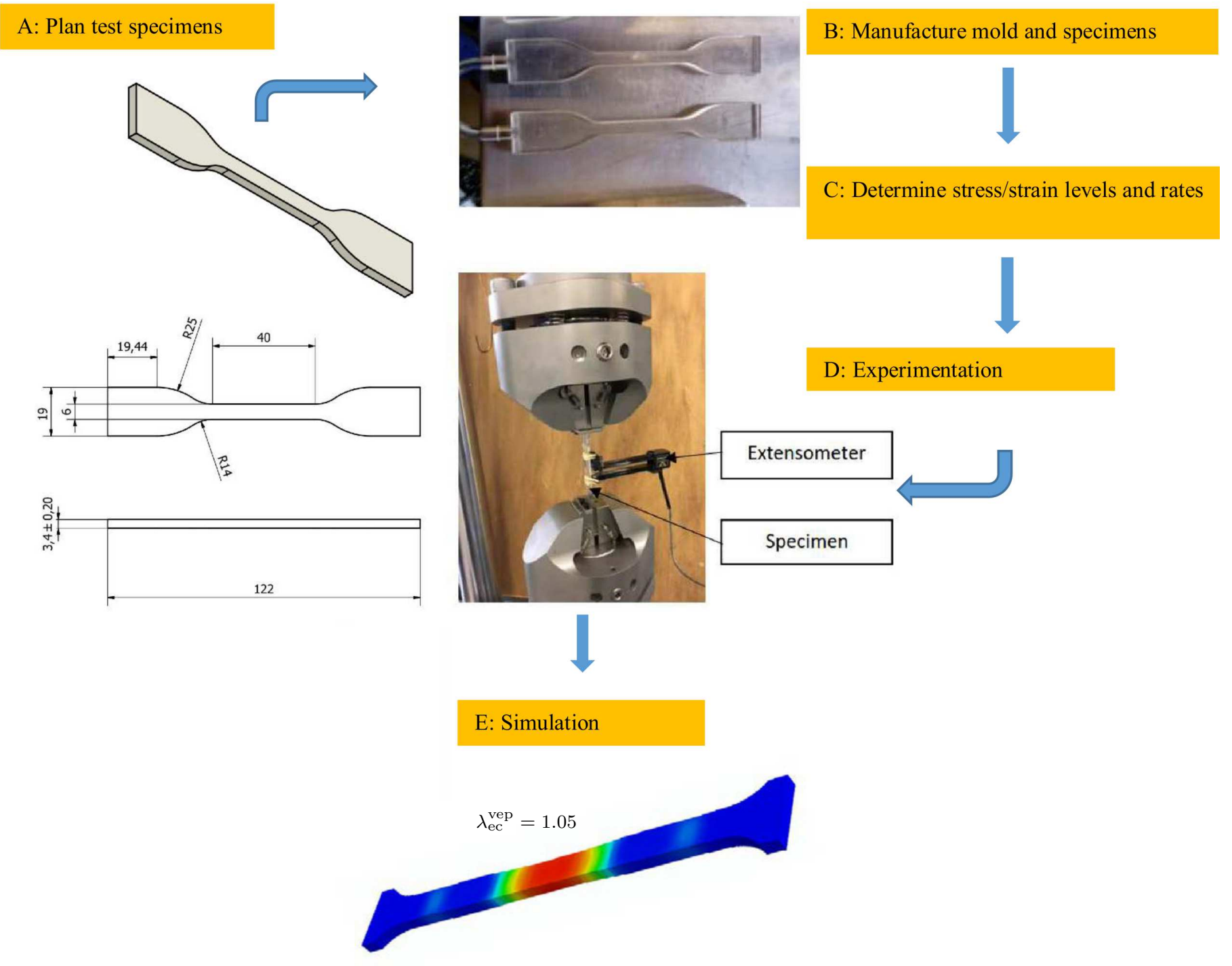

Figure 3: Flowchart for the test program comprising design of the test specimens, uniaxial tensile test set up, and simulations.

The force-controlled cyclic tests were conducted under sinusoidal wave form ranging between the maximum stress and minimum stress based on different stress ratios of $R=0.1$ and $R=0.5$ (the ratio between the smallest and largest stresses). The values of maximum stresses investigated were $97 \%, 90 \%, 75$ $\%$, and $50 \%$ of the peak yield stress, $60 \mathrm{MPa}$. The data acquisition frequency for cyclic tests was $1000 \mathrm{~Hz}$ while the loading frequency was $f=5 \mathrm{~Hz}$, i.e. 200 points per each cycle were recorded. At least two specimens were tested at each level, and the tests interrupted due to the failure of the specimens. 

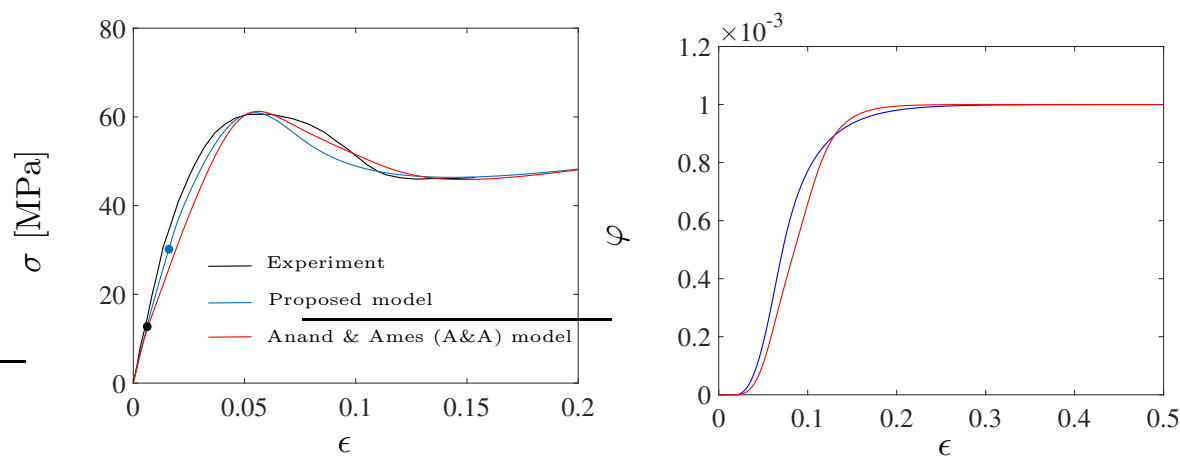

Figure 4: Stress-strain curves (using the final set of material parameters) in tension on $\mathrm{PC}$ at the constant strain rate of $0.001 \mathrm{~s}^{-1}$ (left). The markers indicate the points as the viscoelastic (black circle) and viscoplastic (blue circle) micro-mechanisms (2) and (1) start displaying a notable effect. Evolution of the free-volume $\varphi$ for $\mathrm{PC}$ according to the proposed model (blue curve) and the Anand and Ames (2006)-model (red curve).

For calibration and simulations, the proposed model and peer state-of-theart models were implemented in a computer program using the Intel ${ }^{\circledR}$ Fortran application. The evolution equations used in the numerical solution method are presented in the Appendix B.

\subsection{Model calibration}

In the gauge section of test specimens, uniaxial loading conditions prevailed, and the model reduces to a simplified form facilitating the model calibration. The elastic model parameters were extracted from the strain-controlled, monotonic cold drawing tests. The Young's modulus $E$ was initially calculated from the linear strain range $(0-0.001)$ of the $\sigma-\epsilon$ curve, and then, during calibration, the range was progressively increased ( $E$ was reduced) to sharpen the predictions. The Poisson's ratio $\nu$ typical for solid PC-polymer was found from Holopainen and Wallin (2012); Holopainen (2013). A detailed calibration procedure to find the remaining parameters is discussed next.

Stage 1. The stress-strain response around the peak yield stress and for subsequent softening and hardening at large deformations were first determined under a displacement-controlled monotonic tension by least squares fitting and the data is shown in Fig. 4. Initially, the viscoelastic mechanism (2) was considered constant, i.e. the initial values of both the variables and parameters were applied in (49) and (52). Moreover, $s^{(1)}$ was first considered constant in (41). Then, only the initial flow strength $s_{0}$, the strain-rate-sensitivity parameter $m_{0}$, strain rate parameter $\dot{v}_{0}$, pressure sensitivity parameter $\alpha$, the rubbery modulus $C_{\mathrm{R}}$, and the locking stretch $N$ were needed to be defined. The initial parameters $C_{\mathrm{R}}$ and $N$ governing anisotropic hardening at large srains were taken from Boyce et al. (1989) (for a PC). Other initial values were taken from (Anand and Ames, 2006, p. 1168) (for a PMMA-polymer).

Stage 2. Continuing with data given in Fig. 4, the deformation resistance $s^{(1)}$ was allowed to evolve along with the free volume $\varphi$ when the parameters $h_{0}, g_{0}, b, s_{\mathrm{cv}}$, and $\varphi_{\mathrm{cv}}$ were calibrated. Assuming that the change of $\varphi$ is significantly small, the value $\varphi_{\mathrm{cv}}=0.001$ proposed in Anand and Ames (2006) was 

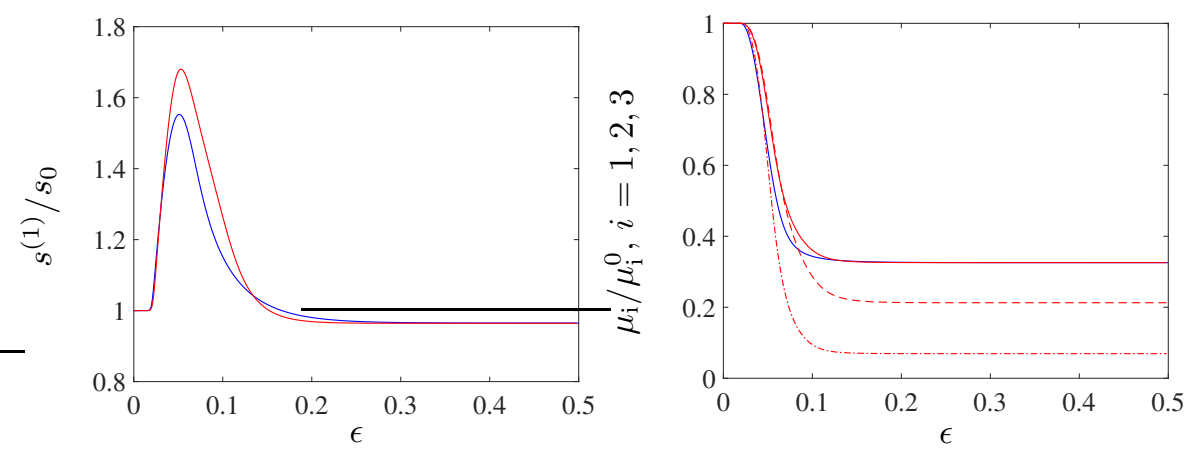

Figure 5: Evolution of the shear resistance $s^{(1)}$ for PC according to the proposed (blue curve) and the Anand and Ames (2006)-model (red curve) based on the final set of calibrated parameters. Corresponding evolutions of the stiffness $\mu_{1}$ (left). For the Anand and Ames (2006)-model, also the stiffnesses $\mu_{2}$ (dashed curve) and $\mu_{3}$ (dashedand-dotted curve) for additional viscoelastic micro-mechanisms are shown.

chosen. The parameter $h_{0}$ directly influences the pre-peak slope, whereas the resting parameters influence the peak yield stress and its post-peak slope, and were determined by the least squares fitting. The initial values of the parameters were extracted from (Anand and Ames, 2006, p. 1168). The parameters determined in this stage have only a minor effect to the ones that had already been determined in the previous stage for large deformations.

The development of the free volume $\varphi$ and the deformation resistance $s^{(1)}$ with strain is depicted in Fig. 4. The comparison of Fig. 4(right) with the macroscopic stress-strain response in Fig. 4(left) (at strain values of $0.03-0.13$ ) reveals that the deformation resistance influenced by the free volume is the one that strongly influences the shape of the yield peak.

Stage 3. The remaining parameters for viscoelasticity $\left(m_{1}, s^{(2)}, c_{1}, \mu_{1, \text { sat }}\right.$, $\left.\mu_{1}^{0}\right)$ were finally defined. Fig. 4 schematically shows the point, in terms of $s^{(2)}$, as the viscoelastic micromechanism approximately starts taking effect. The point at which the viscoplastic deformations start governing the deformation behavior (in terms of $s^{(1)}$ ) has higher strain. Thus, an inspection of Fig. 4 indicates that the initial values of these deformation resistances should range between 10 $30 \mathrm{MPa}$, and satisfy $s^{(1)}>s^{(2)}$. The slope that connects these two points is governed by the modulus $\mu_{1}$ and is, based on the experimentally observed $\sigma-\epsilon$ curves, greater than the elastic modulus $E$ for the initial response (strains 0 0.012). The evolution of $\mu_{1}$ is shown in Fig. 5. The parameters $c_{1}$ and $\mu_{1, \text { sat }}$ in (52) define the slope of softening and the final value of $\mu_{1}$, respectively.

To attain finer details of the experimentally observed stress-strain responses including unloadings, the remaining parameter $m_{1}$ was defined based on the assumption $m_{0}<m_{1}$. Only a few ad hoc iterations were needed to obtain its reasonable value.

The final set of model parameters is given in Tables 2 and 3. For comparison, the Anand and Ames (2006)-model parameters for the tested PC have been determined, and the values are presented in Tables 4, 5, and 6. Moreover, a 
comparison is made with the Jiang et al. (2015)-model that is a straightforward extension of the Anand and Ames (2006)-model. Its additional parameters are presented in Table 7. Compared to the Anand and Ames (2006)-model and Jiang et al. (2015)-model, each requiring 28 and 34 material parameters, only 19 parameters are required in the proposed model.

Table 2: Elastic and viscoelastic model parameters for a PC adapted to a cold drawing test and a cyclic test using the proposed model.

\begin{tabular}{lccccccccc}
\hline \hline Parameter & $E$ & $\nu$ & $\dot{v}_{0}$ & $\alpha$ & $s^{(2)}$ & $m_{1}$ & $c_{1} \cdot 10^{-6}$ & $\mu_{1, \text { sat }}$ & $\mu_{1}^{0}$ \\
\hline Unit ......... & $\mathrm{MPa}$ & & $s^{-1}$ & & $\mathrm{MPa}$ & & $\mathrm{MPa}$ & $\mathrm{MPa}$ & $\mathrm{MPa}$ \\
Value ........ & 2100 & 0.37 & 0.038 & 0.204 & 11 & 0.19 & 4.5 & 1100 & 3500 \\
\hline \hline
\end{tabular}

\section{Evaluation of the approach}

\subsection{Displacement-control}

Based on the final set of parameters, the stress versus strain responses up to the large strain are shown in Fig. 4. For comparison, the Anand and Ames (2006)-model response is also shown. Despite a fairly reduced parameter set, the proposed model captures the experimental response well. A low post-peak slope observed in the experimental response right after the peak yield stress is due to an inhomogeneous three-axial deformation behavior exhibiting short-term necking (strain increases at almost a constant stress level).

The capability of the model in predicting the strain rate dependence is demonstrated in Fig. 6. The model can predict the increasing peak yield stresses with increasing strain rates well. At the highest strain rate, the test specimens showed a brittle fracture, immediately when the peak yield stress was reached. Further, other experimental responses up to this strain value are presented in Fig. 6. Because the model predictions represent a single material (integration) point without failure, the model responses are able to replicate significant softening followed by hardening at large strains.

Table 3: Viscoplastic parameters using the proposed model $(\varphi(0)=0)$.

\begin{tabular}{lccccccccc}
\hline \hline Parameter & $s_{0}$ & $m_{0}$ & $C_{\mathrm{R}}$ & $N$ & $h_{0}$ & $g_{0}$ & $b$ & $s_{\mathrm{cv}}$ & $\varphi_{\mathrm{cv}}$ \\
\hline Unit ......... & $\mathrm{MPa}$ & & $\mathrm{MPa}$ & & $\mathrm{MPa}$ & & & $\mathrm{MPa}$ & \\
Value ........ & 28.5 & 0.055 & 14.0 & 1.65 & 4500 & 0.017 & 755 & 27.5 & 0.001 \\
\hline \hline
\end{tabular}


Table 4: Elastic and viscoelastic parameters for the PC using the Anand and Ames (2006)-model. Moreover, $m_{2}=m_{3}=m_{1}$.

\begin{tabular}{lcccccccc}
\hline \hline Parameter & $E$ & $\nu$ & $\dot{v}_{0}$ & $\alpha$ & $s^{(1)}$ & $s^{(2)}$ & $s^{(3)}$ & $m_{1}$ \\
\hline Unit ......... & $\mathrm{MPa}$ & & $s^{-1}$ & & $\mathrm{MPa}$ & $\mathrm{MPa}$ & $\mathrm{MPa}$ & \\
Value ......... & 2100 & 0.37 & 0.038 & 0.204 & 11 & 17 & 21 & 0.19 \\
\hline \hline
\end{tabular}

\subsection{Load-control}

A creep-recovery test was first conducted because during cyclic loadings a part of the accumulated deformation comes from creep, and also the capacity to predict recovery implicates the capacity to predict cyclic response. The isochronous test comprises the elastic domain (the first load-unload), viscoelastic domain (second load-unload), and the viscoelastic-plastic domain (third loadunload), see Fig. 7, ASTM Committee (2001). As it has been shown, the proposed model captures the creep strains (at nonzero constant stress levels) and subsequent recovery at zero-stress fairly well. Although the model seems to exaggerate the irreversible plastic strain, the value of the residual strain at the end of the test is small.

Figure 8 shows the predicted and experimentally observed hysteresis loops,

Table 5: Viscoelastic model parameters using the Anand and Ames (2006)-model (continued).

\begin{tabular}{lccccccccc}
\hline \hline Parameter & $c_{1} \cdot 10^{-6}$ & $c_{2} \cdot 10^{-6}$ & $c_{3} \cdot 10^{-6}$ & $\mu_{1, \text { sat }}$ & $\mu_{2, \text { sat }}$ & $\mu_{3, \text { sat }}$ & $\mu_{1}^{0}$ & $\mu_{2}^{0}$ & $\mu_{3}^{0}$ \\
\hline Unit ........ & $\mathrm{MPa}$ & $\mathrm{MPa}$ & $\mathrm{MPa}$ & $\mathrm{MPa}$ & $\mathrm{MPa}$ & $\mathrm{MPa}$ & $\mathrm{MPa}$ & $\mathrm{MPa}$ & $\mathrm{MPa}$ \\
Value ......... & 4.5 & 1.8 & 1.3 & 1100 & 600 & 250 & 3500 & 3500 & 3900 \\
\hline \hline
\end{tabular}

Table 6: Viscoplastic parameters for the PC using the Anand and Ames (2006)-model $(\varphi(0)=0)$.

\begin{tabular}{lcccccccccc}
\hline \hline Parameter & $s_{0}$ & $m_{0}$ & $C_{\mathrm{R}}$ & $N$ & $h_{0}$ & $g_{0}$ & $b$ & $s_{\mathrm{cv}}$ & $\varphi_{\mathrm{cv}}$ & $c_{0} \cdot 10^{-6}$ \\
\hline Unit $\ldots \ldots \ldots$ & $\mathrm{MPa}$ & & $\mathrm{MPa}$ & & $\mathrm{MPa}$ & & & $\mathrm{MPa}$ & $\mathrm{MPa}$ \\
Value $\ldots \ldots \ldots . .$. & 28.0 & 0.055 & 14.0 & 1.65 & 16500 & 0.028 & 860 & 27.0 & 0.001 & 0 \\
\hline \hline
\end{tabular}

Table 7: Additional viscoelastic constitutive model parameters for the PC using the Jiang et al. (2015)-model.

\begin{tabular}{lcccccc}
\hline \hline Parameter & $s_{1, \text { sat }}$ & $s_{2, \text { sat }}$ & $s_{3, \text { sat }}$ & $d^{1}$ & $d^{2}$ & $d^{3}$ \\
\hline Unit $\ldots . . . . .$. & $\mathrm{MPa}$ & $\mathrm{MPa}$ & $\mathrm{MPa}$ & $\mathrm{MPa}$ & $\mathrm{MPa}$ & $\mathrm{MPa}$ \\
Value $\ldots . . . . .$. & 40.0 & 40.0 & 40.0 & 75.0 & 60.0 & 50.0 \\
\hline \hline
\end{tabular}



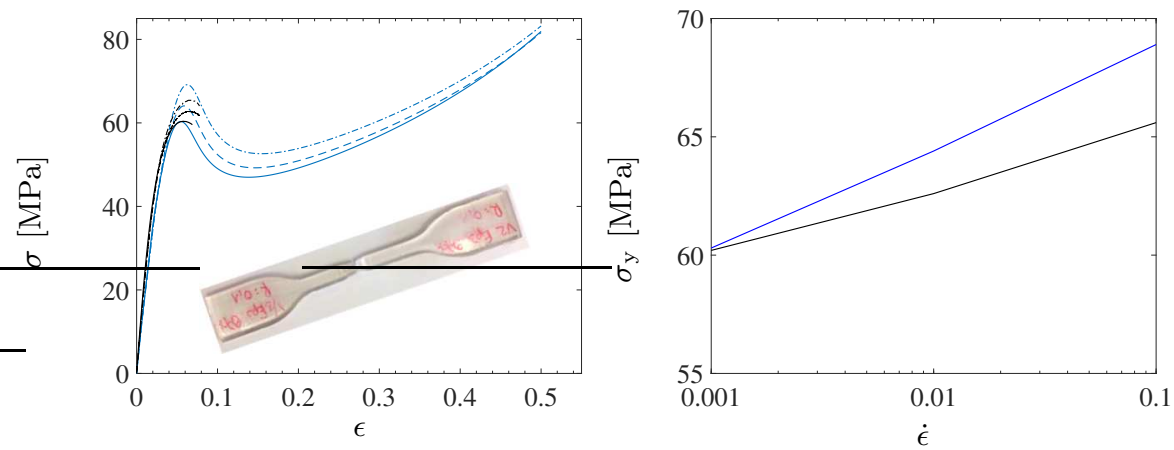

Figure 6: Experimental tensile stress-strain curves on PC at constant strain rates of $0.001 s^{-1}$ (black solid curve), $0.01 s^{-1}$ (black dashed curve), and $0.1 s^{-1}$ (black dashand-dot curve) (left). The blue colored curves implicate the model responses. Peak yield stress vs strain rate in tension (right).
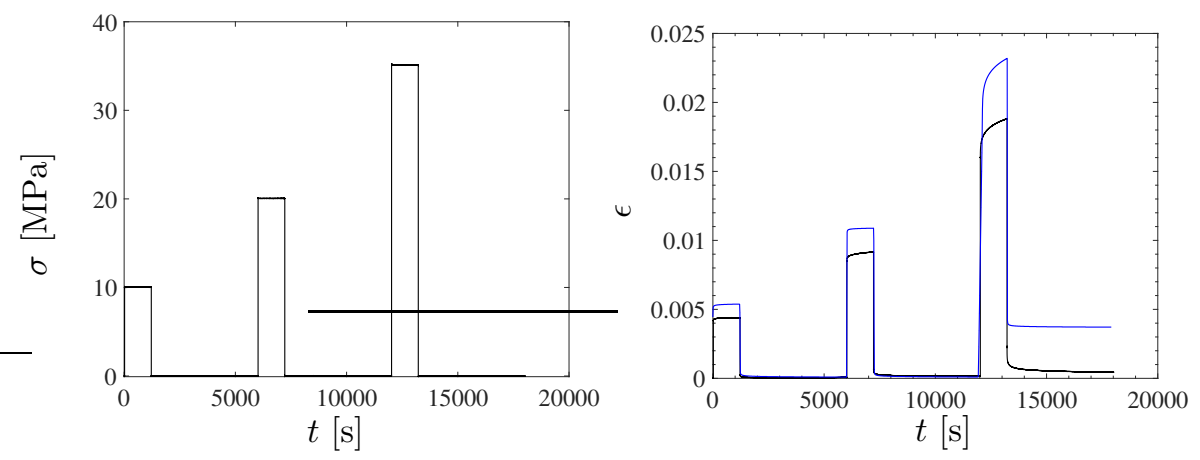

Figure 7: Loading for the creep-recovery test (left). The corresponding strain responses according to the experiment (black) and the proposed model (blue) (right).

as the maximum stress is right below the peak yield stress. Despite the reduced set of material parameters, comparison of the responses reveals that the proposed model is even more accurate than the Anand and Ames (2006)-model. However, due to a large number of parameters needed in the Anand and Ames (2006)-model, the optimum set of calibrated parameters is difficult to find. It can also be observed that the incorporation of only one viscoelastic micromechanism in the proposed model makes possible the nonlinear loading and unloading responses with acceptable accuracy. This characteristic is due to the evolution of $\mu_{1}$ shown in Fig. 5 that results in a suitable material stiffness which is primarily responsible for slightly increasing stiffness of the initial loading response as well as the nonlinear unloading behavior, Anand and Ames (2006).

For comparison, the prediction by the Boyce et al. (1989)-model is also demonstrated in Fig. 8. Owing to the linear elastic constitutive description without viscoelasticity, Boyce et al. (1989)-model is not able to predict the nonlinear hysteresis loops or ratcheting under the applied maximum stress.

The influence of the loading frequency, predicted by the proposed model, 

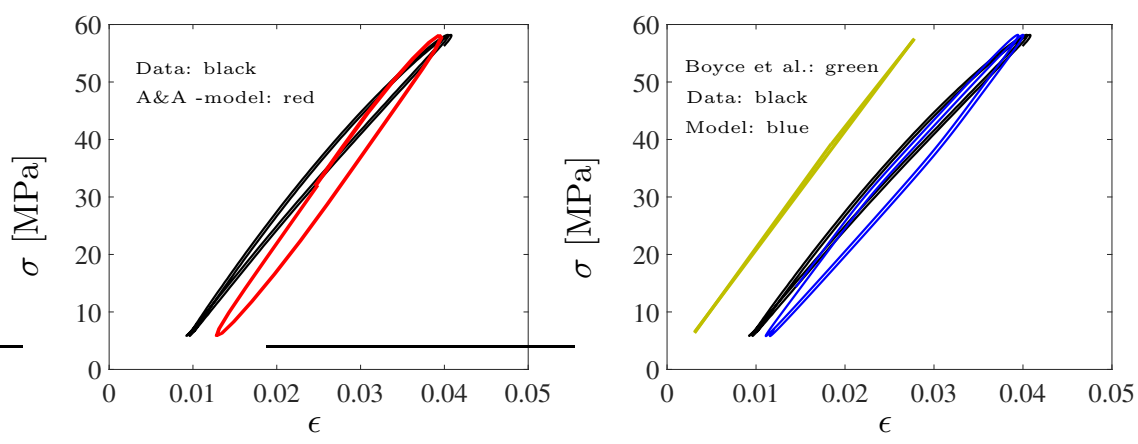

Figure 8: First hysteresis loops of the Anand and Ames (2006)-model vs data with the stress ratio $R=0.1$ and the frequency $f=5 \mathrm{~Hz}$ (left). Comparison of the loops by the proposed model, data, and Boyce et al. (1989)-model predictions (right).
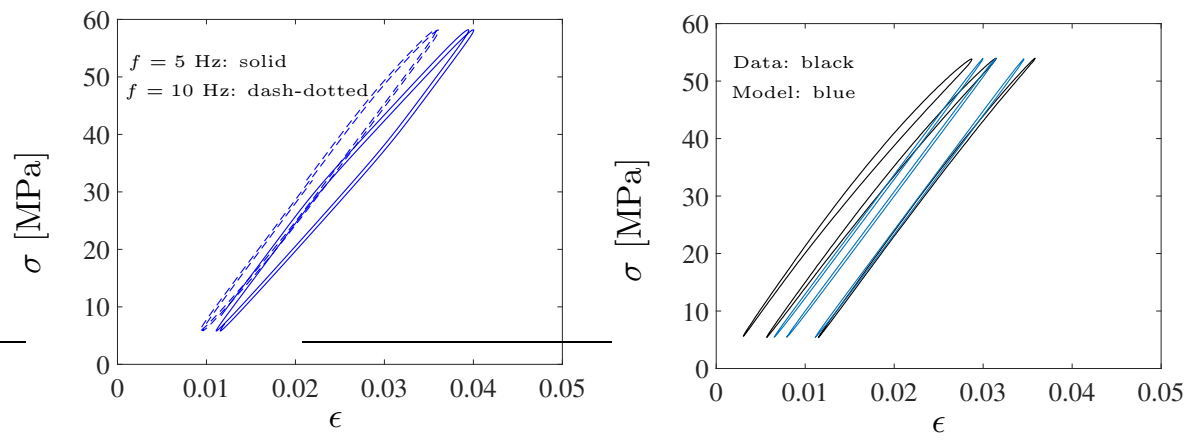

Figure 9: First hysteresis loops by the proposed model with $R=0.1$ and different loading frequencies (left). 30th, 300th, and 2500th loop when $f=5 \mathrm{~Hz}$ (right).

is demonstrated in Fig. 9(left). The material shows hardening, i.e. stretching reduces apparently with increasing loading frequency. This behavior is typical for many amorphous polymers, Boyce et al. (1989); Anand and Ames (2006).

The selected loops under lower maximum stress values are depicted in Figures 9(right) and 10. Hysteresis loops exhibit ratcheting (also termed cyclic creep), i.e. the mean strain grows as the applied load varies between its maximum and minimum values, cf. Jiang et al. (2015); Holopainen et al. (2017); Kang and Kan (2017). A significant initial increase of ratcheting deformation is due to the great mean stress level used. Then the growth of mean strain gradually reduces during the fluctuating loading, which characteristic can be termed as cyclic hardening. In our test specimens, cyclic hardening was recognized as a slightly inhomogeneous deformation behavior (macro-scopically manifested as initial necking), i.e. stretch initially increased at fixed maximum/minimum tensional stress levels, but rapidly attained its stabilized final value.

To demonstrate the ratcheting behavior more precisely, the ratcheting (mean) 

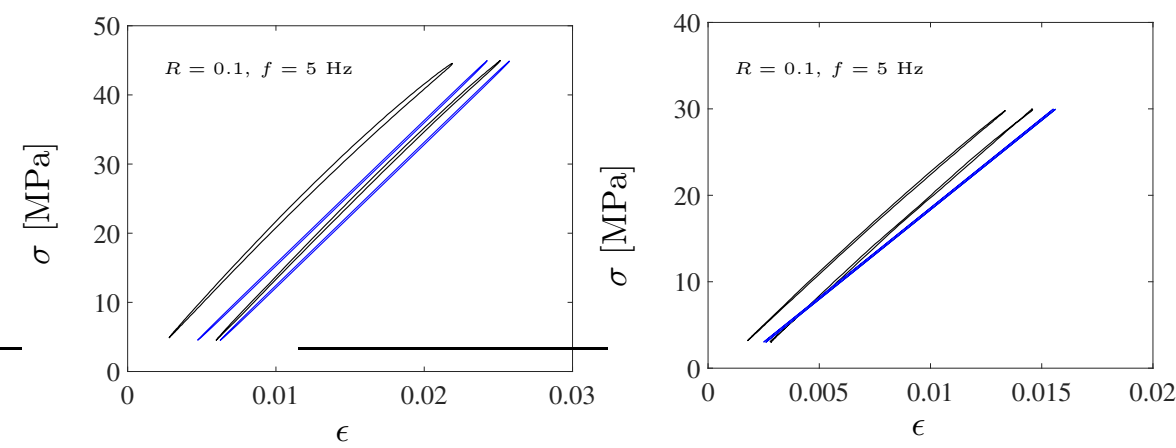

Figure 10: 30th and 7000th hysteresis loops. The black and blue colors imply the experimental data and predicted results according to the proposed model.
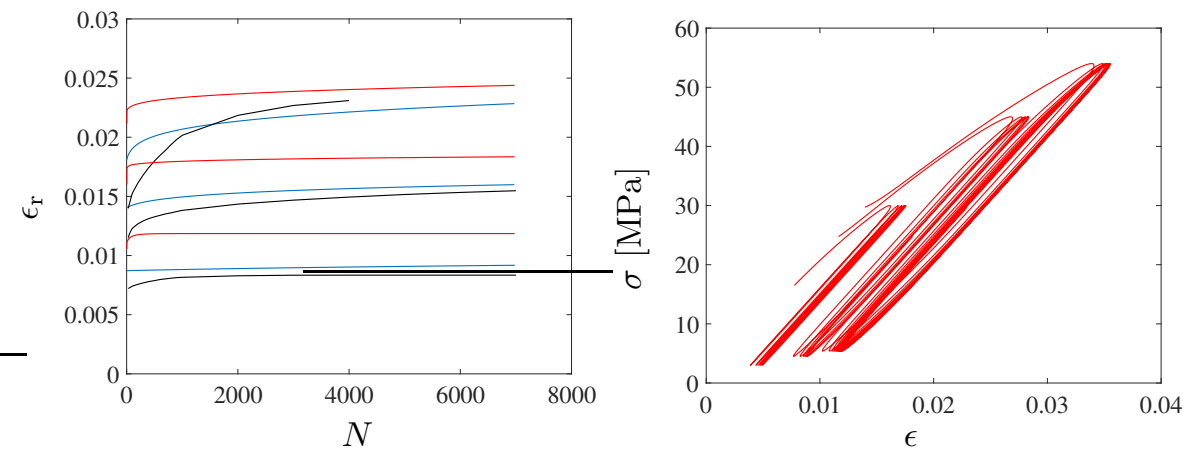

Figure 11: Ratcheting strain responses with $R=0.1$ and the frequency $f=5 \mathrm{~Hz}$. The black, blue, and red colors imply the experimental data and predicted results according to the proposed model and the Anand and Ames (2006)-model, respectively (left). Corresponding hysteresis loops by the Anand and Ames (2006)-model (right).

strain is defined by:

$$
\epsilon_{\mathrm{r}}:=\frac{1}{2}\left(\epsilon_{\min }+\epsilon_{\max }\right)
$$

wherein $\epsilon_{\min }$ and $\epsilon_{\max }$ mean the minimum strain and the maximum strain in each cycle.

Development of $\epsilon_{\mathrm{r}}$ is demonstrated in Fig. 11(left). Under the high mean stresses applied, the proposed model slightly overestimate the ratcheting strain as the number of cycles $N<2000$. Considering the highest maximum stress, the model response also seems to differ from the experimental curve in the intermediate region as $N=2000-6000$. The difference in this region is due to an experimentally observed temperature peak resulting in slightly nonisothermal conditions and thus, increased ratcheting strain, Fig. 12. Otherwise, the temperature increase remains low, and the proposed model well captures the experimentally observed ratcheting deformation at different stress levels.

Using the parameters given in Tables 4, 5, and 6, Anand and Ames (2006)model slightly overestimates the strains at minimum stress values as shown in Fig. 8 and thus, also overpredicts the ratcheting strains demonstrated in Fig. 11. Using the additional material parameters for Jiang et al. (2015)-model in 

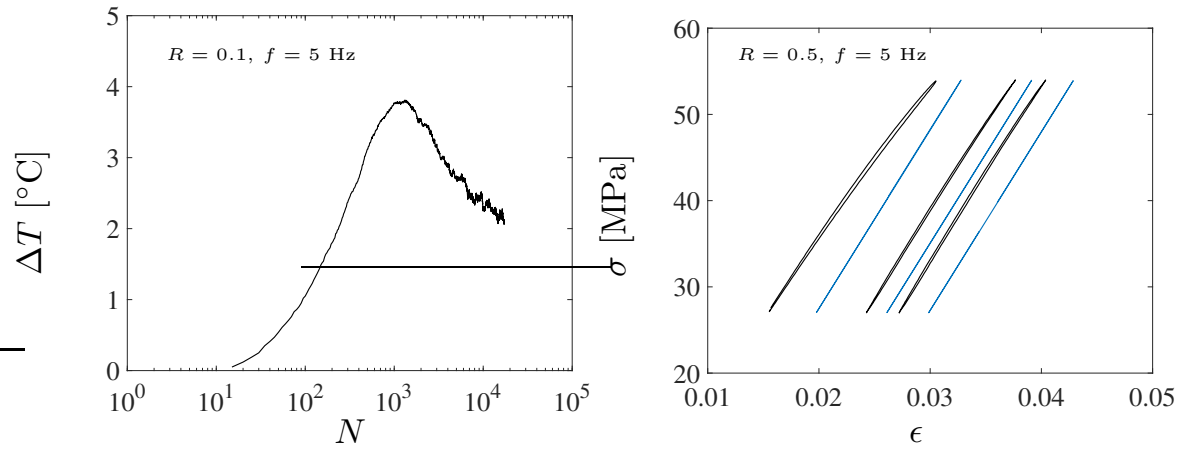

Figure 12: The temperature rise during the loading as the maximum stress is $54 \mathrm{MPa}$ (left). 30th, 3000th, and 10,000th hysteresis loops (right). The black and blue colors imply the experimental data and predicted results according to the proposed model.
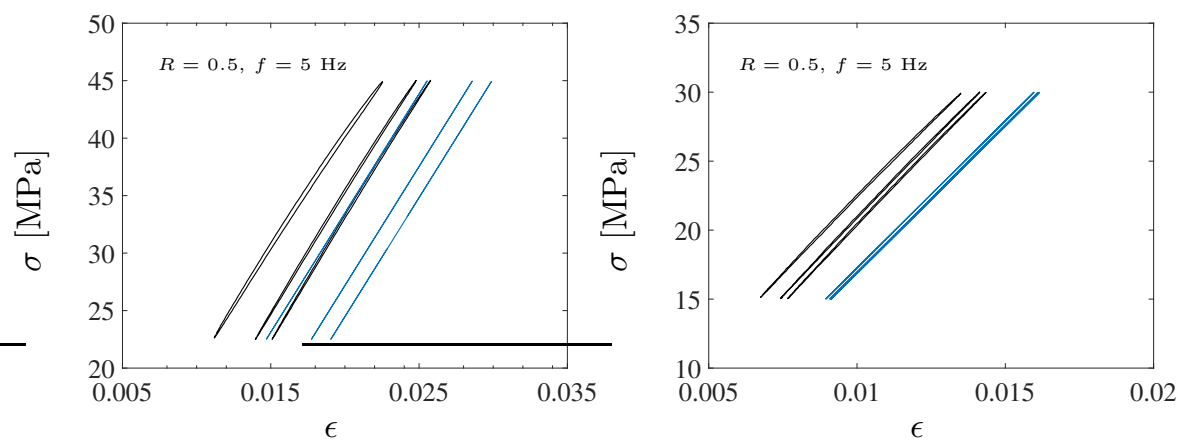

Figure 13: 30th, 3000th, and 10,000th hysteresis loops. The black and blue colors imply the experimental data and predicted results according to the proposed model.

Table 7, no difference with the Anand and Ames (2006)-model response was observed, cf. (Jiang et al., 2015, Fig. 5).

The loops and the development of the ratcheting strain under different maximum stresses for $R=0.5$ are demonstrated in Figs 12, 13, and 14, respectively. Because the temperature now does not influence the mechanical behavior (the rice remains under $5{ }^{\circ} \mathrm{C}$ ), the proposed model is able to predict the ratcheting strain with reasonable accuracy, especially when compared to the Anand and Ames (2006)-model, Fig. 14.

\section{Concluding remarks and further research avenues}

The article introduces a compact viscoelastic-viscoplastic constitutive model for solid polymers capable of improving the predictions of the cyclic deformation behavior at stress levels below the peak yield stress. It is also evidenced that the model is accurate under very large strains, as monotonic loadings are applied. The proposed model is based on the celebrated Haward and Thack- 

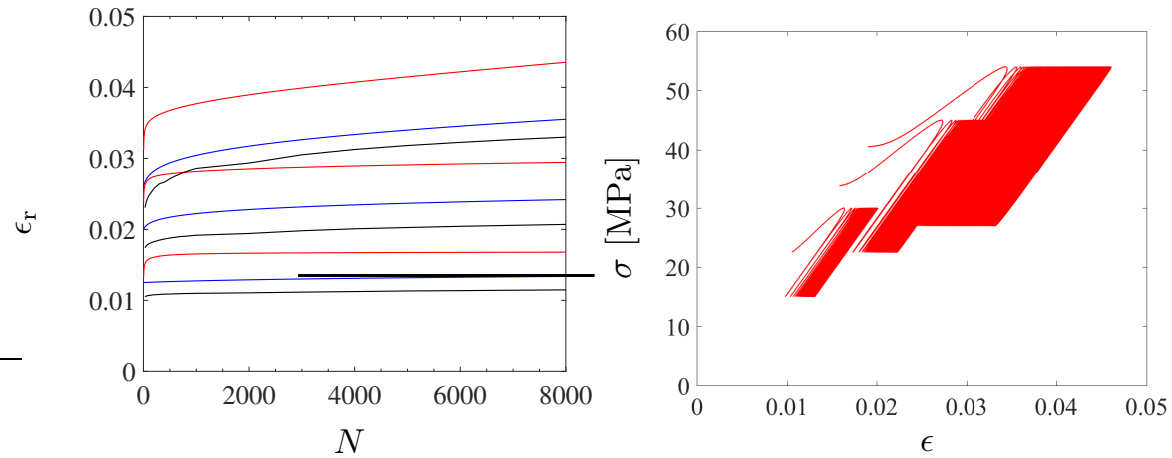

Figure 14: Ratcheting strain responses with the stress ratio $R=0.5$. The colors black and blue imply the experimental data and predicted results according to the proposed model (left). The Anand and Ames (2006)-model predictions are also shown (red color). Corresponding predictions of the hysteresis loops by the Anand and Ames (2006)-model (right). The loops up to 3000 cycles have been shown.

ray (1968)-model and its three dimensional extension by Boyce et al. (1989) for glassy polymers being augmented by a few thermodynamically motivated internal state variables able to predict the missing viscous deformation behavior (correct unloading and ratcheting behavior) under cyclic loadings. Against the distinguished models by Anand and Ames (2006); Jiang et al. (2015) that also are able to predict cyclic deformation behavior well, the proposed model possesses the following advantages:

- a reduced set of internal state variables and material parameters need to be defined;

- elastic portion of the deformation is clearly described by a linear spring separate to viscoelastic-plastic elements according to the classical Haward and Thackray (1968) -model;

- the nonlinear Langevin spring is defined solely using the viscous (viscoelasticplastic) deformation in accordance with the micromechanics-based Boyce et al. (1989)-model;

- purely plastic and viscoelastic deformation are defined by the specified stress controlling the corresponding micromechanisms.

From a number of uniaxial tension experiments, it is shown that this compact formulation, in combination with the calibration procedure can reasonably predict the shape of the hysteresis loops and long-term ratcheting behavior. The comparison with Anand and Ames (2006); Jiang et al. (2015) -models indicates that, despite the larger number of parameters needed in these distinguished models, the proposed model is at least as accurate. Creep-recovery tests evidenced also the capability of the model. Due to the compact formulation, the proposed model is easy to implement and can be used as a built-in feature in different finite-element packages.

Although the uniaxial experimentation has been comprehensive, the proposed model needs to be evaluated under cyclic shear/torsion and arbitrary multidimensional loadings. It should be noted that the research concerning the experimentation of multi-axial cyclic plasticity is practically nonexistent in lit- 
erature. Considering the high loading rates, the effect of temperature rises and needs to be encompassed in the model. Furthermore, an important problem is the modeling of fatigue damage (incl. microstructural changes) that supposedly develops under the applied load levels.

\section{Acknowledgements}

The authors acknowledge the technician Ly Phanousith from FEMTO-ST institute for conducting all the experiments and sharing his expertise in the field of measurements. We want to show also thanks to Professor Jean-Luc Bouvard (Mines-Paris Tech., France) for his valuable comments.

\section{Appendix A. Dissipation inequality vs specific constitutive equations}

Using (33) in (21) for the dissipation inequality, yields

$$
\mathcal{D}=\boldsymbol{\tau}_{\mathrm{A}}: \boldsymbol{d}^{\mathrm{vep}}-\boldsymbol{\beta}^{\mathrm{ve}}: \boldsymbol{d}^{\mathrm{ve}}-\frac{\partial \psi^{\mathrm{ve}}}{\partial \varphi} \dot{\varphi}
$$

which needs to be positive or zero. Using the function (50), $\partial \psi^{\mathrm{ve}} / \partial \varphi \dot{\varphi}=$ $\frac{3}{2} \dot{\mu}_{1}\left(\left(\lambda_{\mathrm{ec}}^{\mathrm{ve}}\right)^{2}-1\right)^{6}$. Since $\dot{\mu}_{1}$ is negative or zero (see (52) and Fig. 5$),\left(\left(\lambda_{\mathrm{ec}}^{\mathrm{ve}}\right)^{2}-1\right)$ must be positive or zero in order to get $-\partial \psi^{\mathrm{ve}} / \partial \varphi \dot{\varphi} \geq 0$. Using (51) for $\lambda_{\mathrm{ec}}^{\mathrm{ve}}$, gives trace $\left(\overline{\boldsymbol{C}}^{\mathrm{ve}}\right) \geq 3$. It can be shown (using principal stretches and noting incompressibility, $\operatorname{det}\left(\overline{\boldsymbol{C}}^{\mathrm{ve}}\right)=1$ by (40)) that this condition is always valid.

It remains to show that $\boldsymbol{\tau}_{\mathrm{A}}: \boldsymbol{d}^{\mathrm{vep}}-\boldsymbol{\beta}^{\mathrm{ve}}: \boldsymbol{d}^{\mathrm{ve}} \geq 0$. Using (37), (38), and (39),

$\boldsymbol{\tau}_{\mathrm{A}}: \boldsymbol{d}^{\mathrm{vep}}-\boldsymbol{\beta}^{\mathrm{ve}}: \boldsymbol{d}^{\mathrm{ve}}=\sqrt{2} \tau^{(1)}\left(\dot{\gamma}^{\mathrm{vp}}+\dot{\gamma}^{\mathrm{ve}} \frac{\tau^{(1)}}{\tau^{(2)}}\right)-\frac{\dot{\gamma}^{\mathrm{ve}}}{\sqrt{2} \tau^{(2)}}\left[\operatorname{tr}\left(\boldsymbol{\beta}^{\mathrm{ve}}\left(\boldsymbol{\tau}^{\mathrm{A}}-\boldsymbol{\beta}^{\mathrm{ve}}\right)\right)+\operatorname{tr}\left(\boldsymbol{\tau}^{\mathrm{A}} \boldsymbol{\beta}^{\mathrm{ve}}\right)\right]$

where the abbreviation tr means trace. Since $\dot{\gamma}^{\mathrm{vp}}, \dot{\gamma}^{\mathrm{ve}}, \tau^{(1)}$, and $\tau^{(2)}$ are positive, it is sufficient to show that the last term in (A.1) is less than the sum of other terms. Since trace $\left(\boldsymbol{\tau}^{\mathrm{A}} \boldsymbol{\beta}^{\mathrm{ve}}\right) \leq \operatorname{trace}\left(\left(\boldsymbol{\tau}^{\mathrm{A}}\right)^{2}\right)=2\left(\tau^{(1)}\right)^{2}$,

$$
\frac{\dot{\gamma}^{\mathrm{ve}}}{\sqrt{2} \tau^{(2)}}\left[\operatorname{tr}\left(\left(\boldsymbol{\beta}^{\mathrm{ve}}\right)\left(\boldsymbol{\tau}^{\mathrm{A}}-\boldsymbol{\beta}^{\mathrm{ve}}\right)\right)+\operatorname{tr}\left(\boldsymbol{\tau}^{\mathrm{A}} \boldsymbol{\beta}^{\mathrm{ve}}\right)\right] \leq \sqrt{2} \dot{\gamma}^{\mathrm{ve}} \frac{\left(\tau^{(1)}\right)^{2}}{\tau^{(2)}}
$$

which is less than the sum of other terms on r.h.s of (A.1).

\section{Appendix B. Numerical solution method}

System of the equations

To define the intermediate placement uniquely, $\boldsymbol{F}^{\mathrm{e}}$ is regarded as symmetric, see Boyce et al. (1989); Holopainen and Wallin (2012). This implies that the visco-plastic spin $\overline{\boldsymbol{W}}^{\mathrm{vp}}$ is generally nonzero. This restriction is adjusted by

\footnotetext{
${ }^{6}$ Evolution of the viscoelastic stretching $\dot{\overline{\boldsymbol{C}}}^{\text {ve }}$ (and thus, $\overline{\boldsymbol{C}}^{\text {ve }}$ ) is considered independent on the free volume $\varphi$ which is meant for the viscoplastic stretching in accordance with its evolution law (44). Actually, change in the free-energy due to the free-volume change can be shown to be small, cf. (Anand and Ames, 2006, p. 1159).
} 
proposing a spin $\tilde{\boldsymbol{W}}^{\text {vep }}$ which is algorithmically consistent, i.e. it becomes finally skew-symmetric at the end of the integration step, Holopainen and Wallin (2012); Engqvist et al. (2016); Holopainen et al. (2017). Then, supposing the current deformation gradient $\boldsymbol{F}$ is known (as it does in standard finite-element implementations), the variables needed to be solve become:

$$
\boldsymbol{Y}:=\left[\boldsymbol{F}^{\mathrm{e}} \overline{\boldsymbol{C}}^{\mathrm{ve}} \tilde{\boldsymbol{W}}^{\mathrm{vp}} s^{(1)} \mu_{1} \varphi\right] .
$$

The deformation measure $\overline{\boldsymbol{C}}^{\text {ve }}$ defines the backstress needed in the flow-type rule (38) and its evolution is defined in (27). Moreover, the viscoelastic spin is considered zero. Consistent with (27), but noting the evolution of the viscoplastic deformation can include a spin, the evolution of $\overline{\boldsymbol{C}}^{\text {vp }}$ can be defined by $\dot{\overline{\boldsymbol{C}}}^{\mathrm{vp}}:=\overline{\boldsymbol{L}}^{\mathrm{vp}} \overline{\boldsymbol{C}}^{\mathrm{vp}}+\overline{\boldsymbol{C}}^{\mathrm{vp}} \overline{\boldsymbol{L}}^{\mathrm{vp}, \mathrm{T}}$. However, this strain measure is optional, and only used for post plotting purposes, see (39) for viscoplastic flow. Once $\boldsymbol{F}^{\mathrm{e}}$ is known, $\boldsymbol{F}^{\text {vep }}$ is solved from the decomposition (1).

Based on an implicit Euler scheme, the exponential update of $\boldsymbol{F}^{\mathrm{vep}}$ in accordance with Miehe et al. (1993) is used, i.e.

$$
\boldsymbol{F}^{\mathrm{vep}}=\exp \left(\Delta t \tilde{\boldsymbol{L}}^{\mathrm{vep}}\right) \boldsymbol{F}_{n}^{\mathrm{vep}},
$$

where $\tilde{\boldsymbol{L}}^{\text {vep }}=\overline{\boldsymbol{D}}^{\mathrm{vep}}+\tilde{\boldsymbol{W}}^{\mathrm{vp}}$. To simplify notation, $n+1$ for indicating the updated state is omitted, whereas $n$ is used to refer to the known state $t_{n}$.

To evaluate $\tilde{\boldsymbol{W}}^{\mathrm{vp}}$, its skew-symmetry-property as well as the symmetry of $\boldsymbol{F}^{\mathrm{e}}$ at the end of the integration interval are used. Finally, the following equations must to be solved:

$$
\begin{aligned}
\boldsymbol{R}_{1} & :=\boldsymbol{F}^{\mathrm{e}}-\boldsymbol{F}_{n}^{\mathrm{vep}-1} \exp \left(-\Delta t \tilde{\boldsymbol{L}}^{\mathrm{vep}}\right), \\
\boldsymbol{R}_{2} & :=\overline{\boldsymbol{C}}^{\mathrm{ve}}-\overline{\boldsymbol{C}}_{\mathrm{n}}^{\mathrm{ve}}-\overline{\boldsymbol{D}}^{\mathrm{ve}} \overline{\boldsymbol{C}}^{\mathrm{ve}}-\overline{\boldsymbol{C}}^{\mathrm{ve}} \overline{\boldsymbol{D}}^{\mathrm{ve}}, \\
\boldsymbol{R}_{3} & :=\operatorname{skew}\left(\boldsymbol{F}^{\mathrm{e}}\right), \\
\boldsymbol{R}_{4} & :=\operatorname{sym}\left(\tilde{\boldsymbol{W}}^{\mathrm{vp}}\right), \\
R_{5} & :=\left(s^{(1)}-s_{\mathrm{n}}^{(1)}-\Delta t \dot{s}^{(1)}\right) / s_{0}, \\
R_{6} & :=\left(\mu_{1}-\mu_{1, \mathrm{n}}-\Delta t \dot{\mu}_{1}\right) / \mu_{1}^{0}, \\
R_{7} & :=\varphi-\varphi_{n}-\Delta t \dot{\varphi},
\end{aligned}
$$

where the first system $\boldsymbol{R}_{1}$ encases (B.2) in the decomposition (1). The residuals $\boldsymbol{R}_{2}$ and $\boldsymbol{R}_{4}$ in (B.3) consist of six equations and $\boldsymbol{R}_{3}$ of three equations (27 state variables in all).

Solution of (B.3) is obtaioned by the Newton-Raphson method. The solution becomes $\boldsymbol{Y}_{\mathrm{n}+1}=\boldsymbol{Y}_{\mathrm{n}}+\Delta \boldsymbol{Y}$, where

$$
\Delta \boldsymbol{Y}=-\boldsymbol{J}^{-1} \boldsymbol{R}, \quad \boldsymbol{J}:=\frac{\partial \boldsymbol{R}}{\partial \boldsymbol{Y}}(\text { Jacobian }), \boldsymbol{R}:=\left[\begin{array}{lllllll}
\boldsymbol{R}_{1} & \boldsymbol{R}_{2} & \boldsymbol{R}_{3} & \boldsymbol{R}_{4} & R_{5} & R_{6} & R_{7}
\end{array}\right]
$$

The system (B.3) is well suitable for standard finite-element codes, Holopainen and Wallin (2012); Engqvist et al. (2016). Therein, the algorithmic tangent stiffness (ATS) tensor is of major importance. The procedure to obtain the ATS tensor can be found from (Holopainen and Wallin, 2012, p. 8). An initial simulation result based on the system (B.3) is shown in Fig. 3 . 


\section{Reduction to uniaxial stress}

The evolution equations for uniaxial loadings used in the calibration and simulations are defined below. The total deformation gradient under such conditions is expressed by:

$$
\boldsymbol{F}=v_{11} \boldsymbol{e}_{1} \otimes \boldsymbol{e}_{1}+v_{22} \boldsymbol{e}_{2} \otimes \boldsymbol{e}_{2}+v_{33} \boldsymbol{e}_{3} \otimes \boldsymbol{e}_{3},
$$

where $\left\{\boldsymbol{e}_{i}, i=1,2,3\right\}$ are the unit base vectors and $v_{i i}=F_{i i}$ denote the principal stretches having the relation $J=\operatorname{det}(\boldsymbol{F})=v_{11} v_{22}^{2}>0$ with the rotation $\boldsymbol{R}$ being unity. The only nonzero stress component is given by:

$$
\sigma_{11}=\frac{1}{J} E \ln \left(v_{11}^{\mathrm{e}}\right)
$$

where $E$ is the Youngs modulus and $v_{11}^{\mathrm{e}}$ is the principal stretch of $\boldsymbol{v}^{\mathrm{e}}$ defined in (2). Owing to the uniaxial stress state, the elastic deformation is constrained by

$$
\ln \left(v_{33}^{\mathrm{e}}\right)=\ln \left(v_{22}^{\mathrm{e}}\right)=-\nu \ln \left(v_{11}^{\mathrm{e}}\right)
$$

where $\nu$ is the Poissons ratio. Due to the symmetry, $v_{33}=v_{22}$ in (B.5), when it follows from the decomposition (1) and (B.7) that $F_{33}^{\mathrm{vep}}=F_{22}^{\mathrm{vep}}$. Since $\boldsymbol{F}^{\mathrm{vep}}$ and $\boldsymbol{F}^{\mathrm{e}}$ are diagonal, $\bar{C}_{33}^{\text {vep }}=\bar{C}_{22}^{\text {vep }}$ in (3). Then, noting volume-preserving viscoelastic-plastic deformation, the relation

$$
\bar{C}_{22}^{\mathrm{vep}}=\left(F_{22}^{\mathrm{vep}}\right)^{2}=1 / F_{11}^{\mathrm{vep}}
$$

holds. Similarly, based on (40) also $\bar{C}_{33}^{\mathrm{vp}}=\bar{C}_{22}^{\mathrm{vp}}=1 / \sqrt{\bar{C}_{11}^{\mathrm{vp}}}$ and $\bar{C}_{33}^{\mathrm{ve}}=\bar{C}_{22}^{\mathrm{ve}}=$ $1 / \sqrt{C_{11}^{\mathrm{ve}}}$ in (5) are isochoric strains.

Because the uniaxial stress state is inherently spin less and results in $\boldsymbol{F}^{\mathrm{e}}$ being symmetric, the tensor valued equations in the system (B.3) reduces to

$$
\begin{aligned}
& R_{1}:=\bar{C}_{11}^{\mathrm{vep}}-\bar{C}_{\mathrm{n}, 11}^{\mathrm{vep}}-2 \bar{D}_{11}^{\mathrm{vep}} \bar{C}_{11}^{\mathrm{vep}}, \\
& R_{2}:=\bar{C}_{11}^{\mathrm{ve}}-\bar{C}_{\mathrm{n}, 11}^{\mathrm{ve}}-2 \bar{D}_{11}^{\mathrm{ve}} \bar{C}_{11}^{\mathrm{ve}} .
\end{aligned}
$$

Considering displacement-control: once $\bar{C}_{11}^{\mathrm{vep}}$ and thus, $F_{11}^{\mathrm{vep}}$ are known, $F_{11}^{\mathrm{e}}$ can be solved from the decomposition (1) during iterations, i.e. $F_{11}^{\mathrm{e}}=F_{11} / F_{11}^{\mathrm{vep}}$. When force-control is applied, $F_{11}^{\mathrm{e}}=v_{11}^{\mathrm{e}}$ is directly available from the constitutive equation (B.6). Then, once $\bar{C}_{11}^{\mathrm{vep}}$ and thus, $F_{11}^{\mathrm{vep}}$ are known, $F_{11}$ is available from the decomposition (1).

\section{References}

Anand, L., Ames, N.M., 2006. On modeling the micro-indentation response of an amorphous polymer. Int. J. Plasticity 22, 1123-1170.

Anand, L., Gurtin, M.E., 2003. A theory of amorphous solids undergoing large deformations with application to polymer glasses. Int. J. Solids Structures 40, 1465-1487.

ASTM Committee, 2001. ASTM D2990-01, Standard test methods for tensile, compressive, and flexural creep and creep- rupture of plastics. ASTM International , 1-20.

ASTM Committee, 2003. Designation: D 638 - 03. Standard test method for tensile properties of plastics. ASTM International , 1-15. 
Awaja, F., Zhang, S., Tripathi, M., Nikiforov, A., Pugno, N., 2016. Cracks, microcracks and fracture in polymer structures: Formation, detection, autonomic repair. Progress in Materials Science 83, 536-573.

Barriere, T., Cheng, G., Holopainen, S., 2018. Modeling, simulation, and experimentation of fatigue behavior in amorphous solids. Key Engineering Materials 774, 210-216.

Beesley, R., Chen, H., Hughes, M., 2017. A novel simulation for the design of a low cycle fatigue experimental testing programme. Comp. Struct. 178, 105-118.

Belytschko, T., Liu, W.K., Moran, B., 2000. Nonlinear Finite Elements for Continua and Structures. John Wiley \& Sons, Chichester.

Bengström, J., 2015. Mechanics of Solid Polymers: Theory and Computational Modeling (1st edition). Elsevier.

Bouvard, J.L., Ward, D.K., Hossain, D., Marin, E.B., Bammann, D.J., Horstemeyer, M.F., 2010. A general inelastic internal state variable model for amorphous glassy polymers. Acta Mech 213, 71-96.

Boyce, M.C., Weber, G.G., Parks, D.M., 1989. On the kinematics of finite strain plasticity. J. Mech. Phys. Solids 37, 647-665.

Chaboche, J.L., 1997. Thermodynamic formulation of constitutive equations and application to the viscoplasticity and viscoelasticity of metals and polymers. Int. J. Solids Structures 34, 2239-2254.

Cohen, A., 1991. A pade approximant to the inverse langevin function. Rheol Acta 30, $270-273$.

Deng, Y.J., Peng, L.F., Lai, X.M., Fu, M.W., Lin, Z.Q., 2017. Constitutive modeling of size effect on deformation behaviors of amorphous polymers in micro-scaled deformation. Int. J. Plasticity 89, 197-222.

Engqvist, J., Wallin, M., Ristinmaa, M., Hall, S.A., Plivelic, T.S., 2016. Modelling multi-scale deformation of amorphous glassy polymers with experimentally motivated evolution of the microstructure. J. Mech. Phys. Solids 96, 497-510.

George, M., Nziakou, Y., Goerke, S., Genix, A.C., Ciccotti, M., 2018. In situ AFM investigation of slow crack propagation mechanisms in a glassy polymer. J. Mech. Phys. Solids 112, $109-125$.

Gudimetla, M.R., Doghri, I., 2017. A finite strain thermodynamically-based constitutive framework coupling viscoelasticity and viscoplasticity with application to glassy polymers. Int. J. Plasticity 98, 197-216.

Haward, R.N., Thackray, G., 1968. The use of a mathematical model to describe isothermal stress-strain curves in glassy thermoplastics. Proc. Roy. Soc. A. 302, 453-472.

Holopainen, S., 2013. Modeling of the mechanical behavior of amorphous glassy polymers under variable loadings and comparison with state-of-the-art model predictions. Mechanics of Materials 66, 35-58.

Holopainen, S., Barriere, T., 2018. Modeling of mechanical behavior of amorphous solids undergoing fatigue loadings, with application to polymers. Computers and Structures 199, $57-73$.

Holopainen, S., Barriere, T., Cheng, G., Kouhia, R., 2017. Continuum approach for modeling fatigue in amorphous glassy polymers. applications to the investigation of damageratcheting interaction in polycarbonate. Int. J. Plasticity 91, 109-133. 
Holopainen, S., Wallin, M., 2012. Modeling of long-term behavior of amorphous glassy polymers. ASME J. Engng. Materials Technol. 135, 1-11.

Hughes, J.M., Lugo, M., Bouvard, J.L., McIntyre, T., Horstemeyer, M.F., 2017. Cyclic behavior and modeling of small fatigue cracks of a polycarbonate polymer. Int. J. Fatigue $99,78-86$

James, M.N., Lu, Y., Christopher, C.J., Patterson, E.A., 2013. Crack path support for deformation mechanisms in fatigue of polycarbonate. Engng. Fract. Mech. 108, 89-97.

Jiang, C.K., Jiang, H., Zhang, J.W., Kang, G.Z., 2015. A viscoelasticplastic constitutive model for uniaxial ratcheting behaviors of polycarbonate. Polymer Engineering And Science 55, 2559-65.

Jiang, H., Zhang, J., Yang, Z., Jiang, C., Kang, G., 2017. Modeling of competition between shear yielding and crazing in amorphous polymers scratch. Int. J. Solids Structures 124, $215-228$.

Kang, G., Kan, Q., 2017. Cyclic Plasticity of Engineering Materials: Experiments and Models. John Wiley \& Sons, Chichester.

Krairi, A., Doghri, I., 2014. A thermodynamically-based constitutive model for thermoplastic polymers coupling viscoelasticity, viscoplasticity and ductile damage. Int. J. Plasticity 60, $163-181$.

Miehe, C., Stein, E., Wagner, W., 1993. Associative multiplicative elasto-plasticity formulation and aspects of the numerical implementation including stability analysis. Comp. Struct. $52,969-978$.

Qi, Z., Hu, N., Li, G., Zeng, D., Su, X., 2019. Constitutive modeling for the elastic-viscoplastic behavior of high density polyethylene under cyclic loading. Int. J. Plasticity 113, 125-144.

Ravi Chandran, K.S., 2016. Mechanical fatigue of polymers: A new approach to characterize the S-N behavior on the basis of macroscopic crack growth mechanism. Polymer 91, 222238.

Ritchie, R.O., 1999. Mechanisms of fatigue-crack propagation in ductile and brittle solids. Int. J. Fracture 100, 55-83.

Shojaei, A.K., Volgers, P., 2018. A coupled hyperelastic-plastic-continuum damage model for studying cyclic behavior of unfilled engineering polymers. Int. J. Fatigue 107, 33-39.

Talamini, B., Mao, Y., Anand, L., 2018. Progressive damage and rupture in polymers. J. Mech. Phys. Solids 111, 434-457.

Tomita, Y., Uchida, M., 2003. Characterization of micro- to macroscopic deformation behavior of amorphous polymer with heterogeneous distribution of microstructures. Int. J. Mech. Sci. $45,1703-1716$.

Wu, P.D., Van der Giessen, E., 1993. On improved network models for rubber elasticity and their applications to orientation hardening in glassy polymers. J. Mech. Phys. Solids 41, $427-456$.

Zienkiewicz, O.C., Taylor, R.L., Fox, D.D., 2014. The Finite Element Method for Solid and Structural Mechanics (7th edition). Elsevier. 